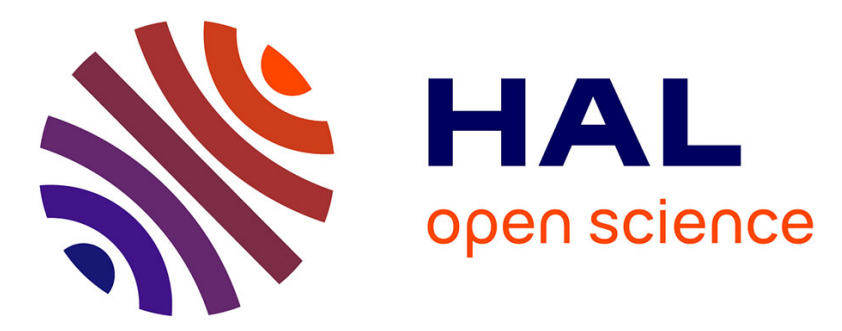

\title{
Consistent formulation for the Discrete-Continuous Model: Improving complex dislocation dynamics simulations
}

\author{
O. Jamond, R. Gatti, Arjen Roos, Benoit Devincre
}

\section{- To cite this version:}

O. Jamond, R. Gatti, Arjen Roos, Benoit Devincre. Consistent formulation for the DiscreteContinuous Model: Improving complex dislocation dynamics simulations. International Journal of Plasticity, 2016, 80, pp.19 - 37. 10.1016/j.ijplas.2015.12.011 . hal-01637572

\section{HAL Id: hal-01637572 https://hal.science/hal-01637572}

Submitted on 22 Jan 2018

HAL is a multi-disciplinary open access archive for the deposit and dissemination of scientific research documents, whether they are published or not. The documents may come from teaching and research institutions in France or abroad, or from public or private research centers.
L'archive ouverte pluridisciplinaire HAL, est destinée au dépôt et à la diffusion de documents scientifiques de niveau recherche, publiés ou non, émanant des établissements d'enseignement et de recherche français ou étrangers, des laboratoires publics ou privés. 


\begin{abstract}
A new and efficient formulation of the Discrete-Continuous Model (DCM) for the simulation of $3 \mathrm{D}$ dislocation dynamics in complex finite or periodic volumes is presented. As in previous versions, the improved model is based on a coupling between a Dislocation Dynamics (DD) code and a Finite Element (FE) code through eigenstrain theory. Short-range interactions are now handled more properly. Specifically, in the continuous limit the stress field driving the dislocation dynamics is now reconstructed consistently. Furthermore, the DCM can now handle nonstructured meshes, and free surface and interface handling does not depend on having a structured mesh anymore. Also numerical experiments shed some light on the influence of the choice of the FE quadrature. Some approximations are proposed and justified, and the use of advanced algorithmic techniques are used for time integration and the homogeneisation procedure to reach a high computational efficiency. Basic tests demonstrate the validity and the efficiency of the proposed strategy. Remarkably, it is demonstrated that for a periodic domain the
\end{abstract}

\footnotetext{
*Corresponding author: olivier.jamond@ cea.fr. Present address: CEA - Paris Saclay, France.

** Present address: Safran Tech - Paris Saclay, France.
} 
DCM with a very fine FE mesh is actually faster than a corresponding classical DD simulation.

Keywords: Dislocation dynamics, Finite element, Dislocation theory, Crystal plasticity, Micromechanics

\section{Introduction}

Three-dimensional Dislocation Dynamics (DD) simulation has become an essential tool in materials science during the last few years because of the many investigations into the mechanical properties of micro- and nano-objects and because of the need for more physically justified crystal plasticity constitutive rules. In its most standard formulations, DD simulations use analytical expressions for the stress field of dislocation segments which are part of discretised dislocation loops. However those expressions are valid only for isotropic elasticity and in an infinite continuum [1-3]. In order to handle more complex boundary conditions and to take the effects of free surfaces or internal boundaries into account, alternative numerical solutions have also been developed (see for instance [4] for a description and comparison of the main methods). In the following such solutions are called hybrid methods, as opposed to classical methods defined in infinite domains.

Most of the hybrid methods depend on a coupling between DD and Finite Elements (FE). The most widely used approach is the superposition method in which the mechanical problem is decomposed into a DD problem in an infinite medium and a dislocation-free complementary boundary value problem, solved by a FE elastic solver [5-10]. The Peach-Köhler forces driving the dislocation dynamics are then calculated, at line segments, from the stress obtained by adding the stress from the dislocation-free complementary FE problem to the analytical 
stress as calculated in the standard DD simulation in an infinite medium, so that the boundary conditions are taken into account properly. This approach was extensively used and optimised in the past years to simulate mainly plasticity of micro- and nano-objects (see for instance [11-18]).

An alternative hybrid approach is the Discrete-Continuous Model (DCM) [1923]. Here only the short-range dislocation-dislocation interactions are treated analytically and all other interactions including those due to external loads and free boundaries are calculated numerically by FE. Mixed calculations in which the DD responses in one set of regions are combined with phenomenological constitutive laws in other regions of the structure then become almost trivial. The DCM is based on a regularisation of the atomic displacement jump across the slip plane into a plastic strain inclusion following the eigenstrain theory [24]. It was successfully applied to several practical problems, such as anisotropic thin films [25, 26], metal-matrix composites with long fibres [27], micro-pillars [21] and single-crystal $\gamma-\gamma^{\prime}$ Ni based superalloys [28]. Nevertheless, several limitations have appeared in the past years:

- The calculations were restricted to simple geometries due to the need for regular structured meshes made out of hexaedron elements.

- The procedure for regularising slip near domain boundaries was restricted to and optimised for such structured meshes [22].

- Calculations of the dislocation self-stress field at distances around the boundary of the eigenstrain volume suffered from a sharp discontinuity and therefore could be at the origin of an artificial jump in the velocity of two approaching dislocations [29]. 
- The coupling algorithm between the two codes was general and not optimised for the DCM.

In this work, these limitations are addressed through the development of a new formulation for the DCM and through numerical improvements. This new formulation is presented in $\S 2$ and is said to be consistent because it leads to a proper reconstruction of the stress field driving the DD at the continuous limit for the FE problem. Next, numerical optimisations of the DCM procedures are presented in $\S 3$ with some recommendations regarding the choice of the spatial integration in the FE simulation part. The paper is closed with elementary tests presented in $\S 4$ to show the new possibilities and performances of the DCM. Large-scale computation tests with high dislocation densities and complex geometries with associated performance analyses will be presented in a forthcoming paper. In the following, all DCM tests use the microMegas DD simulation code [30] and the Z-set FE solver [31].

\section{The new DCM formulation}

In the DCM, the stress field $\boldsymbol{\sigma}$ driving the movement of the dislocations is a superposition of a stress field $\boldsymbol{\sigma}^{F E}$ computed numerically by the FE code and a local correction stress field $\boldsymbol{\sigma}^{L C}$. The latter is calculated analytically in the vicinity of the dislocation lines in order to reconstruct a pseudo-singular field close to the dislocation lines. The stress $\sigma^{F E}$ transmits the long-range dislocation-dislocation interactions, the body forces and the loads transmitted through the boundary conditions. It takes into account the plastic strains $\boldsymbol{\varepsilon}^{p}$ generated by the movement of dislocations through a regularisation of the displacement jump $[[\boldsymbol{u}]]$ across the slip plane. The regularisation procedure, following the eigenstrain theory [24], intro- 
duces a homogenisation length $h$. The maximum element size of the FE mesh is directly related to this length in order to garuantee that sufficient integration points lie inside each eigenstrain volume. The local correction $\boldsymbol{\sigma}^{L C}$ is computed by the DD code, which then handles the movements of the dislocation lines and their topological contact reactions. These basic principles of the DCM are described in full detail in [22] and schematically illustrated in Fig. 1.

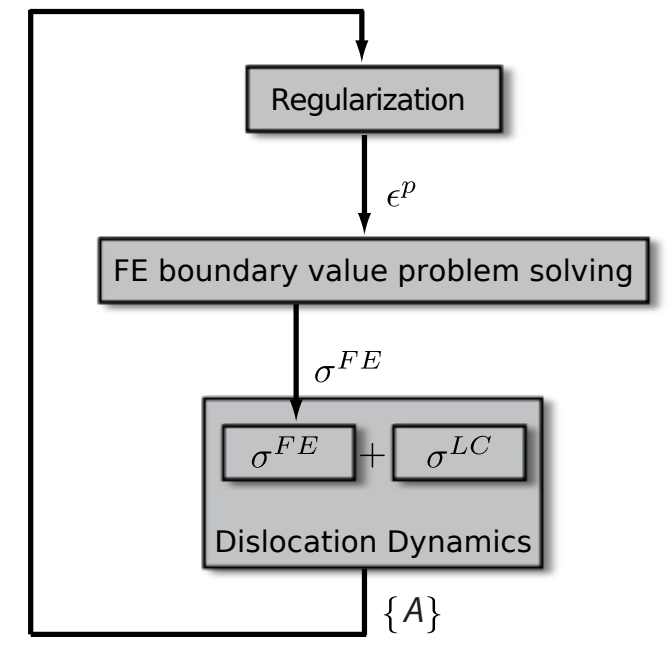

Figure 1: The basic DCM algorithm: the motion of the discrete dislocation lines is handled by the DD simulation code. For the calculation of the Peach-Köhler force, a local correction $\boldsymbol{\sigma}^{L C}$ is calculated internally in the DD code to account for the strong elastic interactions between close segments. The other stress contribution $\boldsymbol{\sigma}^{F E}$ is computed with the FE code when solving the boundary value problem and taking the eigenstrain distribution into account. The latter results from the regularisation of the accumulated slipped areas $\{A\}$.

The calculation of the local correction $\sigma^{L C}$ is a key point of the DCM to reproduce correctly short-distance dislocation-dislocation interactions and contact reactions between dislocation segments. In the previous DCM formulation, $\boldsymbol{\sigma}^{L C}$ is simply the singular stress field $\boldsymbol{\sigma}^{S}$ as is usually used in standard DD simula- 
tions, truncated at a distance $h$ from the dislocation line. This simple solution is precise enough to capture, for instance, the zipping/unzipping process of junctions [22]. However it turns out that for small values of $h$ (i.e., $<50 \mathrm{~nm}$ ), large numerical errors may appear in the dislocation dynamics when dislocation lines are at distances around $h$. This is because the superposition of the two contributions generates a stress discontinuity at the contour of the eigenstrain volume, which becomes significant in those cases.

To overcome this important limitation, a revised local correction is presented in $§ 2.1$. This revised local correction is said to be consistent because at the continuous limit for the FE solution, i.e. at the limit when the element size tends to zero, it leads to an exact reconstruction of the mechanical fields for the problem of an infinite domain. The improved calculation of $\boldsymbol{\sigma}^{L C}$ also implies modifications to the regularisation procedure, i.e. the way in which the plastic eigenstrain $\boldsymbol{\varepsilon}^{p}$ is distributed to the integration points (IP) of the FE mesh. This is presented in $\S 2.2$. Then, the way in which surfaces and interfaces are handled is described in $§ 2.3$.

\subsection{Consistent reconstruction of the stress field}

As for the superposition method, the new DCM formulation is based on the linearity of the problem of finding the displacement and stress fields $(\boldsymbol{u}, \boldsymbol{\sigma})$ in a finite elastic domain $\Omega$ containing displacement jumps $\llbracket \boldsymbol{u} \rrbracket$ due to dislocation glide, and its decomposition into sub-problems which are easier to solve. This reference 
boundary value problem $\mathscr{P}$ can be written as follows:

$\mathscr{P} \mid \begin{array}{ll}\nabla \cdot \boldsymbol{\sigma}+\boldsymbol{f}=\boldsymbol{0} & \text { in } \Omega \backslash\{A\} \\ \boldsymbol{\sigma}=\boldsymbol{E}: \boldsymbol{\varepsilon} & \text { in } \Omega \backslash\{A\} \\ \llbracket \boldsymbol{u} \rrbracket & \text { across }\{A\} \\ \boldsymbol{u}=\boldsymbol{u}_{0} & \text { at } \partial \Omega_{u} \\ \boldsymbol{\sigma} \cdot \boldsymbol{n}=\boldsymbol{t} & \text { at } \partial \Omega_{\sigma} .\end{array}$

The boundary $\partial \Omega$ of $\Omega$ with outward normal $\boldsymbol{n}$ is divided into $\partial \Omega_{u}$ where Dirichlet boundary conditions are applied and into $\partial \Omega_{\sigma}$ (non-overlapping with $\partial \Omega_{u}$ ) where Neumann boundary conditions are applied. At time $t,\{A\}$ represents the area swept by the dislocation loops since the beginning of the simulation. This includes the initial Volterra process used to define the initial eigenstrain distribution associated to the initial dislocation line configuration. The displacement jump $\llbracket \boldsymbol{u} \rrbracket$ is tangent to $\{A\}$, its magnitude and direction are given by the Burgers vector $\boldsymbol{b}$. Furthermore, $\boldsymbol{E}$ is the fourth-order tensor of elasticity, $\boldsymbol{\varepsilon}$ the small-strain strain tensor (the symmetric part of the gradient of the displacement field), $f$ represents the body forces, $\boldsymbol{t}$ the traction applied at Neumann boundaries, and $\boldsymbol{u}_{0}$ is the prescribed displacement at Dirichlet boundaries.

This problem $\mathscr{P}$ can be decomposed linearly into three sub-problems

1. $\mathscr{P} F E$ with solution $\left(\boldsymbol{u}^{F E}, \boldsymbol{\sigma}^{F E}\right)$, where the FE stands for Finite Element even though at this point the problem is not yet discretised, and even though this sub-problem might be solved by any other numerical method,

2. $\mathscr{P}^{S}$ with an analytical Singular $(\mathrm{S})$ solution $\left(\boldsymbol{u}^{S}, \boldsymbol{\sigma}^{S}\right)$, and

3. $\mathscr{P}^{N S}$ with an analytical Non-Singular (NS) solution, written as $\left(\boldsymbol{u}^{N S}, \boldsymbol{\sigma}^{N S}\right)$. 
The three considered sub-problems are

$$
\begin{aligned}
& \mathscr{P} F E=\begin{array}{l}
\nabla \cdot \boldsymbol{\sigma}^{F E}+\boldsymbol{f}=\mathbf{0} \text { in } \Omega \\
\boldsymbol{\sigma}^{F E}=\boldsymbol{E}:\left(\boldsymbol{\varepsilon}^{F E}-\boldsymbol{\varepsilon}^{p}\right) \text { in } \Omega \\
\boldsymbol{u}^{F E}=\boldsymbol{u}_{0}-\left(\boldsymbol{u}^{S}-\boldsymbol{u}^{N S}\right) \text { at } \partial \Omega_{u} \\
\boldsymbol{\sigma}^{F E} \cdot \boldsymbol{n}=\boldsymbol{t}-\left(\boldsymbol{\sigma}^{S}-\boldsymbol{\sigma}^{N S}\right) . \\
\boldsymbol{n} \text { at } \partial \Omega_{\sigma},
\end{array} \\
& \mid \begin{array}{l}
\nabla \cdot \boldsymbol{\sigma}^{S}=\mathbf{0} \text { in } \mathbb{R}^{3} \backslash\{A\} \\
\boldsymbol{\sigma}^{S}=\boldsymbol{E}: \boldsymbol{\varepsilon}^{S} \text { in } \Omega \backslash\{A\} \\
\llbracket \boldsymbol{u}^{S} \rrbracket \text { across }\{A\},
\end{array} \\
& \begin{array}{l|l}
\mathscr{P} N S & \nabla \cdot \boldsymbol{\sigma}^{N S}=\mathbf{0} \text { in } \mathbb{R}^{3} \\
& \boldsymbol{\sigma}^{N S}=\boldsymbol{E}:\left(\boldsymbol{\varepsilon}^{N S}-\boldsymbol{\varepsilon}^{p}\right) \text { in } \Omega .
\end{array}
\end{aligned}
$$

With the definition

$$
(\boldsymbol{u}, \boldsymbol{\sigma}) \equiv\left(\boldsymbol{u}^{F E}, \boldsymbol{\sigma}^{F E}\right)-\left(\boldsymbol{u}^{N S}, \boldsymbol{\sigma}^{N S}\right)+\left(\boldsymbol{u}^{S}, \boldsymbol{\sigma}^{S}\right)
$$

it can be verified that

$$
\mathscr{P}=\mathscr{P}^{F E}-\mathscr{P}^{N S}+\mathscr{P}^{S}
$$

The eigenstrain distribution $\boldsymbol{\varepsilon}^{p}$ is constructed additively over the set of dislocation loops, i.e.

$$
\boldsymbol{\varepsilon}^{p}=\sum_{\ell \in L} \boldsymbol{\varepsilon}_{\ell}^{p}
$$

where $L$ is the set of dislocation loops. The problem $\mathscr{P}^{N S}$ can then be decomposed into dislocation loop-wise sub-problems $\mathscr{P}_{\ell}^{N S}$ :

$$
\mathscr{P}^{N S}=\sum_{\ell \in L} \mathscr{P}_{\ell}^{N S}
$$


where

$$
\begin{array}{l|ll}
\mathscr{P}_{\ell}^{N S} & \nabla \cdot \boldsymbol{\sigma}_{\ell}^{N S}=\mathbf{0} & \text { in } \mathbb{R}^{3} \\
& \boldsymbol{\sigma}_{\ell}^{N S}=\boldsymbol{E}:\left(\boldsymbol{\varepsilon}_{\ell}^{N S}-\boldsymbol{\varepsilon}_{\ell}^{p}\right) & \text { in } \Omega .
\end{array}
$$

Similarly one can write

$$
\llbracket \boldsymbol{u}^{S} \rrbracket=\sum_{\ell \in L} \llbracket \boldsymbol{u}^{S} \rrbracket_{\ell}
$$

and

$$
\mathscr{P}^{S}=\sum_{\ell \in L} \mathscr{P}_{\ell}^{S}
$$

where

$$
\begin{array}{l|ll}
\mathscr{P}_{\ell}^{S} & \nabla \cdot \boldsymbol{\sigma}_{\ell}^{S}=\mathbf{0} & \text { in } \mathbb{R}^{3} \backslash\{A\} \\
\boldsymbol{\sigma}_{\ell}^{S}=\boldsymbol{E}: \boldsymbol{\varepsilon}_{\ell}^{S} & \text { in } \Omega \backslash\{A\} \\
\llbracket \boldsymbol{u}^{S} \rrbracket_{\ell} & \text { across }\{A\} .
\end{array}
$$

The key point of this new DCM decomposition (6) is that one can choose an eigenstrain distribution such that $\boldsymbol{\sigma}_{\ell}^{S}-\boldsymbol{\sigma}_{\ell}^{N S}$ decreases rapidly to zero with increasing distance from the dislocation line $\ell$ (the actual eigenstrain distribution considered is described in $\$ 2.2)$. Thus, the term $\boldsymbol{\sigma}_{\ell}^{S}-\boldsymbol{\sigma}_{\ell}^{N S}$ can be truncated at a given distance $r=r_{l}$ from the dislocation line $\ell$, and the DCM reconstruction of the total stress field then becomes

$$
\begin{aligned}
\boldsymbol{\sigma}^{L C} & \equiv \sum_{\ell \in L} \boldsymbol{\sigma}_{\ell \mid r_{l}}^{S}-\sum_{\ell \in L} \boldsymbol{\sigma}_{\ell}^{N S} \mid r_{l} \\
\boldsymbol{\sigma} & =\boldsymbol{\sigma}^{F E}+\boldsymbol{\sigma}^{L C}
\end{aligned}
$$

where $\boldsymbol{\sigma}_{\ell}^{N S}{ }_{\mid r_{l}}$ and $\boldsymbol{\sigma}_{\ell \mid r_{l}}^{S}$ are the stress fields $\boldsymbol{\sigma}_{\ell}^{N S}$ and $\boldsymbol{\sigma}_{\ell}^{S}$ truncated at a distance $r_{l}$ of the dislocation $\ell$ and the stress $\sigma^{L C}$ is the new definition of the DCM localcorrection term. In what follows, unless noted explicitly, the notations $\boldsymbol{\sigma}^{N S}$ and $\boldsymbol{\sigma}^{S}$ refer to the truncated fields, i.e. $\boldsymbol{\sigma}^{N S}=\sum_{\ell \in L} \boldsymbol{\sigma}_{\ell}^{N S}$ arl and $\boldsymbol{\sigma}^{S}=\sum_{l \in L} \boldsymbol{\sigma}_{\ell \mid r_{l}}^{S}$. 
Remark 1: The difference between this new formulation of the DCM and the superposition method are as follows. The problem $\mathscr{P} F E$ can be decomposed into two sub-problems $\mathscr{P} F E a$ and $\mathscr{P} F E b$ with

$$
\mathscr{P} F E a \mid \begin{aligned}
& \nabla \cdot \boldsymbol{\sigma}^{F E a}+\boldsymbol{f}=\mathbf{0} \text { in } \Omega \\
& \boldsymbol{\sigma}^{F E a}=\boldsymbol{E}: \boldsymbol{\varepsilon}^{F E a} \text { in } \Omega \\
& \boldsymbol{u}^{F E a}=\boldsymbol{u}_{0}-\boldsymbol{u}^{S} \text { at } \partial \Omega_{u} \\
& \boldsymbol{\sigma}^{F E a} \cdot \boldsymbol{n}=\boldsymbol{t}-\boldsymbol{\sigma}^{S} \cdot \boldsymbol{n} \text { at } \partial \Omega_{\sigma}
\end{aligned}
$$

and

$$
\begin{aligned}
& \mathscr{P} F E b \mid \begin{array}{l}
\nabla \cdot \boldsymbol{\sigma}^{F E b}=\mathbf{0} \text { in } \Omega \\
\boldsymbol{\sigma}^{F E b}=\boldsymbol{E}:\left(\boldsymbol{\varepsilon}^{F E b}-\boldsymbol{\varepsilon}^{p}\right) \text { in } \Omega
\end{array} \\
& \boldsymbol{u}^{F E b}=\boldsymbol{u}^{N S} \text { at } \partial \Omega_{u} \\
& \boldsymbol{\sigma}^{F E b} \cdot \boldsymbol{n}=\boldsymbol{\sigma}^{N S} \cdot \boldsymbol{n} \text { at } \partial \Omega_{\sigma} .
\end{aligned}
$$

The superposition method can be written as

$$
\mathscr{P}^{S P}=\mathscr{P}^{F E a}+\mathscr{P}^{S}
$$

and the DCM can be written as

$$
\mathscr{P}=\left(\mathscr{P}^{F E a}+\mathscr{P}^{S}\right)+\mathscr{P}^{F E b}-\mathscr{P}^{N S}
$$

The DCM can then be seen as an extension of the superposition method to which two sub-problems are added. Even though at first sight these two added subproblems may appear as incurring extra computation costs, their introduction can 
actually lead to major computational cost savings with respect to the superposition method and even with respect to classical DD.

In the $\mathscr{P} S P$ problem the dislocation segments interact with each other through problem $\mathscr{P}^{S}$, where each segment generates a slowly decreasing stress field (as $1 / r)$ around its core which cannot be truncated without major loss of accuracy. This leads to a $N$-segment problem, a priori of $O\left(N^{2}\right)$ complexity, in which each dislocation segment interacts with every other one. The a priori here means that some advanced technique such as the Fast Multipole Method (FMM) ([32-34]) might be used to reduce this complexity to $O(N \log (N))$ or ideally to $O(N)$ assuming that the heterogeneity of the dislocation density in the simulated volume can be effectively subdivided into a hierarchical structure.

In the new DCM formulation each dislocation segment interacts with all the other segments through problem $\mathscr{P} F E$, but, thanks to the truncation in equation (13), only with neighbouring segments at $r<r_{l}$ in $\mathscr{P}^{N S}$ and $\mathscr{P} S$. For the latter two problems the complexity a priori then becomes of order $O\left(N \times N_{l}\right)$, where $N_{l}$ is the average number of segments at $r<r_{l}$. Given that $r_{l}$ must be of the order of the element size of the mesh used in problem $\mathscr{P} F E$ (see [22]) and assuming a structured mesh for a cubic simulated volume, $N_{l}$ is of the order $N / n_{\text {elem }}$, with $n_{\text {elem }}$ the number of elements of the mesh. Then the complexity for $\mathscr{P}^{N S}$ and $\mathscr{P}^{S}$ remains $O\left(N^{2}\right)$ but with a prefactor of the order $1 / n_{\text {elem }}$, which can easily reach $10^{-5}$ or $10^{-6}$.

The FE part of the DCM then plays a similar role as the FMM in standard DD simulation codes, because both carry the long-range dislocation-dislocation interactions and thereby significantly reduce the $O\left(N^{2}\right)$ complexity of the problem. In the DCM it has the added advantage of also taking into account the external loads. 
For extremely high segment densities and a large cutoff radius some combination of the two methods might be thought of to reduce the computing load for interactions within the cutoff radius, however, this is not the point of the present paper.

Remark 2: The new DCM decomposition of the stress field $\boldsymbol{\sigma}=\boldsymbol{\sigma}^{F E}-\boldsymbol{\sigma}^{N S}+\boldsymbol{\sigma}^{S}$ can also be interpreted through a different spatial decomposition of the interactions. On the one hand, the stress $\boldsymbol{\sigma}^{R} \equiv \boldsymbol{\sigma}^{F E}-\boldsymbol{\sigma}^{N S}$, which might be called the remote stress field, represents the influence of the external loads and the remote dislocations and other defects including free surfaces and interfaces, that lie at a distance $r>r_{l}$ from the considered point. The stress contribution $\boldsymbol{\sigma}^{N S}$ can then be seen as a filter that removes the influence of the dislocations lying at a distance $r<r_{l}$ from $\sigma^{F E}$. On the other hand, the influence of these nearby dislocations is handled by $\boldsymbol{\sigma}^{S}$.

2.2. Regularisation of the plastic slip and its interpolation with the new eigenstrain distribution

Following the formalism developed in the previous section, the eigenstrain distribution used in the DCM must be such that:

1. it is constructed additively over the set of dislocation loops $L$, i.e. $\boldsymbol{\varepsilon}^{p}=$ $\Sigma_{\ell \in L} \boldsymbol{\varepsilon}_{l}^{p}$,

2. for each $\ell \in L, \boldsymbol{\sigma}_{\ell}^{S}-\boldsymbol{\sigma}_{\ell}^{N S}$ decreases rapidly to zero away from the dislocation line forming the loop $\ell$,

3. one can easily compute the stress field of problem $\mathscr{P}^{N S}$.

It can be noted that such an eigenstrain distribution can be derived exploiting the Burgers vector distribution function proposed by Cai et al. [3] in the context of 
the development of a non-singular continuum theory of dislocations. The eigenstrain distribution, used in the eigenstrain problem $\mathscr{P} F E$, is calculated spreading isotropically the Burgers vector using a specific function denoted $\tilde{w}$. In the following the notation of [3] is used. The analytical expressions for the stress field derived from the Burgers vector distribution function $\tilde{w}$ are not reproduced here. These expressions are needed in the DCM to construct the stress field $\boldsymbol{\sigma}^{N S}$, solution of $\mathscr{P}^{N S}$.

As stated in [3], using $\tilde{w}$ in the FE problem $\mathscr{P}^{F E}$ the stress field $\tilde{\boldsymbol{\sigma}}^{F E}$ is obtained from the resolution of the elasticity problem. The stress field $\tilde{\sigma}^{F E}$ must be convoluted by $\tilde{w}$ in order to get the stress field $\boldsymbol{\sigma}^{F E}$ (see equation (33) of [3]). The main advantage is that with this convolution step the interpolation of the stress field from the IP of the FE mesh to the centres of the dislocation segments can be done without introducing any unwanted smoothing of this field, as was the case in the previous formulations of the DCM. The practical procedure for the construction of the eigenstrain distribution for problem $\mathscr{P}^{F E}$ is now presented in detail.

In the DD part of the DCM, the dislocation segments $i$ glide producing a plane trapezoidal swept area $A_{i}$ (the light grey area shown in Fig. 2a). An elementary dislocation segment $\mathrm{d} \ell$ located at $\boldsymbol{x}_{0}$, gliding a distance $\mathrm{d} y$ in its slip plane, sweeping out an area $\mathrm{d} \boldsymbol{A}=\boldsymbol{n} \mathrm{d} \ell \mathrm{d} y$ (with $\boldsymbol{n}$ its normal), generates an elementary plastic shear distribution $\mathrm{d} \boldsymbol{\varepsilon}^{p}$ given by

$$
\mathrm{d} \boldsymbol{\varepsilon}^{p}(\boldsymbol{x})=\bar{w}_{r_{c}}\left(\left\|\boldsymbol{x}-\boldsymbol{x}_{0}\right\|, h\right) \frac{\boldsymbol{b} \otimes \mathrm{d} \boldsymbol{A}+\mathrm{d} \boldsymbol{A} \otimes \boldsymbol{b}}{2},
$$


(a)

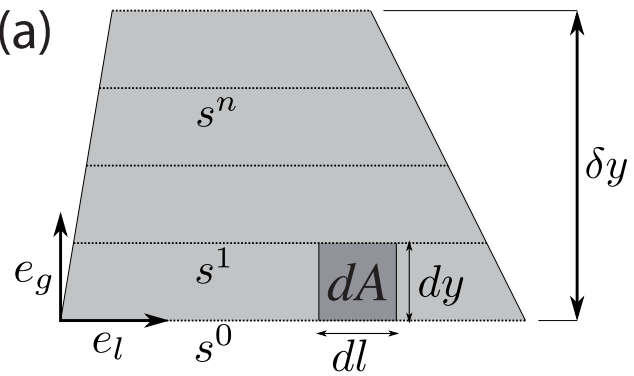

(b)

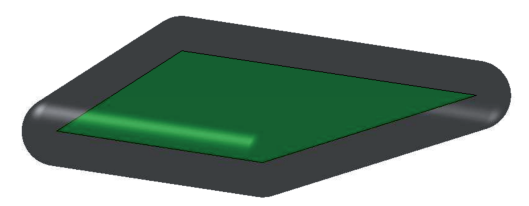

Figure 2: (a) Swept area $A_{i}$ for a single dislocation segment $i$ during a time step. The elementary eigenstrain distribution $\bar{w}_{r_{c}}$ is integrated continuously in the direction $\boldsymbol{e}_{l}$ and discretely in the direction $\boldsymbol{e}_{g}$. The glide of the segment over a length $\delta y$ is decomposed into $n$ glide increments of length $d y$. The $s^{k}$ denote the successive positions of the segment along the $n$ glide increments. (b) Geometry of the inclusion (in grey) generated by the homogenisation of the swept area $A_{i}$ (in green).

where $h$ is the homogenisation length and $\bar{w}_{r_{c}}(r, h)$ is the function $\tilde{w}(r, h)$ of [3] for spreading out the Burgers vector

$$
\begin{aligned}
w(r, h) & =\frac{15 h^{4}}{8 \pi\left(r^{2}+h^{2}\right)^{7 / 2}}, \\
\tilde{w}(r, h) & =0.3425 w(r, 0.9038 h)+0.6575 w(r, 0.5451 h),
\end{aligned}
$$

except that it is truncated at a distance $r=r_{c}$ from $\boldsymbol{x}_{0}$ :

$$
\bar{w}_{r_{c}}(r, h)=\mathscr{H}\left(r_{c}-r\right) \tilde{w}(r, h)
$$

with $\mathscr{H}$ the Heaviside step function.

For saving computational time, $r_{c}$ should be as small as possible, but for too small values the difference between $\bar{w}_{r_{c}}$ and $\tilde{w}$ would lead to loss of a large amount of plastic shear strain. Fig. 3 shows the influence of the cut-off radius $r_{c}$ on the integral over the whole space of $\bar{w}_{r_{c}}$. Its value tends to 1 when $r_{c} \rightarrow \infty$ (i.e. when $\left.\bar{w}_{r_{c}} \rightarrow \tilde{w}\right)$. In the DCM calculations reported in this paper the value $r_{c}=1.75 h$ is 
used, which leads to an error of less than $5 \%$ in the integral of $\bar{w}_{r_{c}}$ with respect to the one of $\tilde{w}$.

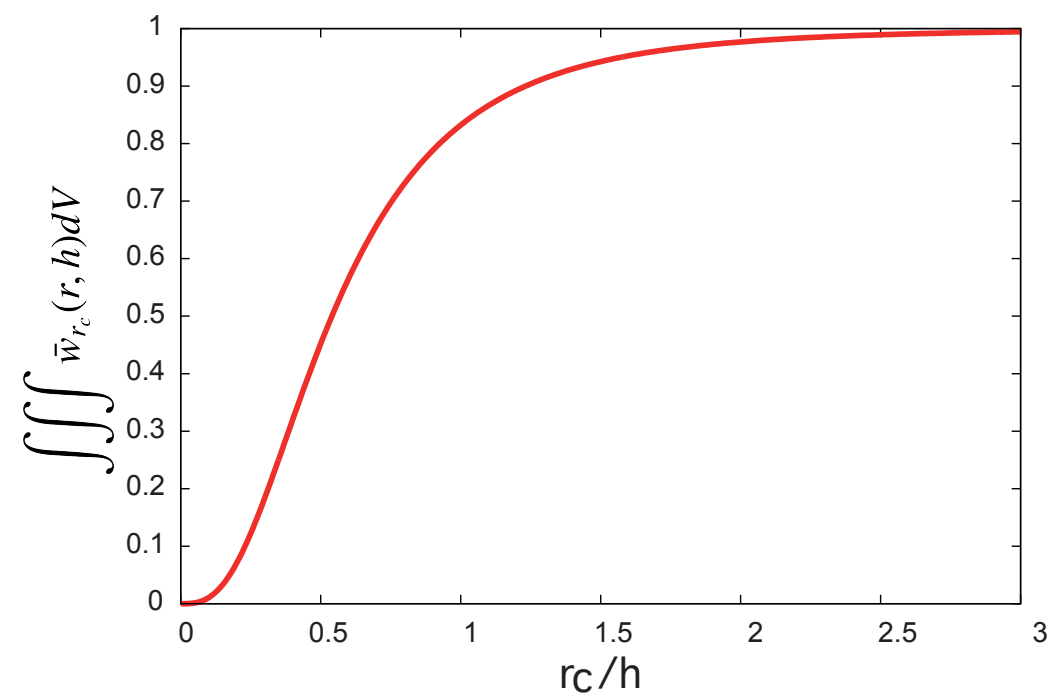

Figure 3: Influence of the cutting radius $r_{c}$ on the value of the integral of $\bar{w}_{r_{c}}$, with $h$ the homogenisation length.

Similarly to the previous version of the DCM (see [22]), the geometry and the distribution of the eigenstrain resulting from a finite swept area is the union of the geometry of, and the eigenstrain associated to the elementary spherical inclusions $\mathrm{d} \Phi_{x_{0}}$ of radius $r_{c}$ associated to each point of the swept area. An example of the resulting shape of the inclusion is shown in Fig. 2b. Then, an IP of the FE mesh at position $\boldsymbol{x}$ gets attributed the sum of the elementary shear of every spherical inclusion $\mathrm{d} \Phi_{x_{0}}$ containing $\boldsymbol{x}$. Formally

$$
\boldsymbol{\varepsilon}^{p}(\boldsymbol{x})=\sum_{A_{i} \in\{A\}} \boldsymbol{e}_{i} \mathscr{I}_{i}(\boldsymbol{x})
$$

with

$$
\mathscr{I}_{i}(\boldsymbol{x}) \equiv \int_{\boldsymbol{x}_{0} \in A_{i}} \bar{w}_{r_{c}}\left(\left\|\boldsymbol{x}-\boldsymbol{x}_{0}\right\|, h\right) \mathrm{d} x_{0}^{2}
$$


where the notation $\mathrm{d} x_{0}^{2}$ denotes a surface integral and $\boldsymbol{e}_{i}=\frac{1}{2}\left(\boldsymbol{b}_{i} \otimes \boldsymbol{n}_{i}+\boldsymbol{n}_{i} \otimes \boldsymbol{b}_{i}\right)$ with $\boldsymbol{b}_{i}$ and $\boldsymbol{n}_{i}$ the Burgers vector and the unit normal vector of the swept area $A_{i}$, respectively. $\{A\}$ is the whole set of swept areas.

In the following part of this paragraph, the subscript $i$ is omitted for clarity. The integral $\mathscr{I}(\boldsymbol{x})$ is integrated continuously in the direction of the dislocation line ( $\boldsymbol{e}_{l}$ in Fig. 2a) and in a discrete way in the glide direction $\left(\boldsymbol{e}_{g}\right.$ in Fig. 2a). In the local reference frame $\left(\boldsymbol{e}_{l}, \boldsymbol{e}_{g}\right)$, the glide distance $\delta y$ along $\boldsymbol{e}_{g}$ during the time step $\delta t$ of a segment $s$ is decomposed into $n$ elementary glides d $y \equiv \delta y / n$. Then

$$
\mathscr{I}(\boldsymbol{x}) \simeq \mathrm{d} y \sum_{k=1}^{n} \int_{\boldsymbol{x}_{0} \in s^{k}} \bar{w}_{r_{c}}\left(\left\|\boldsymbol{x}-\boldsymbol{x}_{0}\right\|, h\right) \mathrm{d} \boldsymbol{x}_{0}, \equiv \mathrm{d} y \sum_{k=1}^{n} \mathscr{J}^{k}(\boldsymbol{x})
$$

where $s^{k}$ refers to the segment $s$ of length $l^{k}$ at position $k$. For the computation of the integral $\mathscr{J}^{k}(\boldsymbol{x})$, the integration and truncation operations are inversed according to

$$
\mathscr{J}^{k}(\boldsymbol{x}) \simeq \mathscr{H}\left(r_{c}-\left\|\boldsymbol{x}-\boldsymbol{x}^{k}\right\|\right) \mathscr{L}^{k}(\boldsymbol{x})
$$

where $\boldsymbol{x}^{k}$ refers to the closest point to $\boldsymbol{x}$ on $s_{k}$ and

$$
\mathscr{L}^{k}(\boldsymbol{x})=\int_{\boldsymbol{x}_{0} \in s^{k}} \tilde{w}_{r_{c}}\left(\left\|\boldsymbol{x}-\boldsymbol{x}_{0}\right\|, h\right) \mathrm{d} \boldsymbol{x}_{0} .
$$

This last integral $\mathscr{L}^{k}(\boldsymbol{x})$ is calculated analytically. To this end, a new local frame $\left(\boldsymbol{C}_{k}, \boldsymbol{e}_{l}, \boldsymbol{e}_{x}^{k}\right)$ is considered, with $\boldsymbol{C}_{k}$ the centre of the segment $s^{k}$ and $\boldsymbol{e}_{x}^{k}$ the unit vector normal to $s^{k}$ and pointing to $x$ (see Fig. 4). With $(x, y)$ the position of $x$ in the local frame, $q=y^{2}+h^{2}, p=x-x_{0}$ :

$$
\mathscr{L}^{k}(x, y)=\left[\frac{h^{4} p\left(15 q^{2}+20 q p^{2}+8 p^{4}\right)}{8 \pi q^{3}\left(q+p^{2}\right)^{5 / 2}}\right]_{p=x_{0}-l^{k} / 2}^{p=x_{0}+l^{k} / 2} .
$$

As stated above, the stress field $\tilde{\boldsymbol{\sigma}}^{F E}$ computed with the FE code at the IP and using the eigenstrain distribution as defined above, must be convoluted with $\tilde{w}$. 


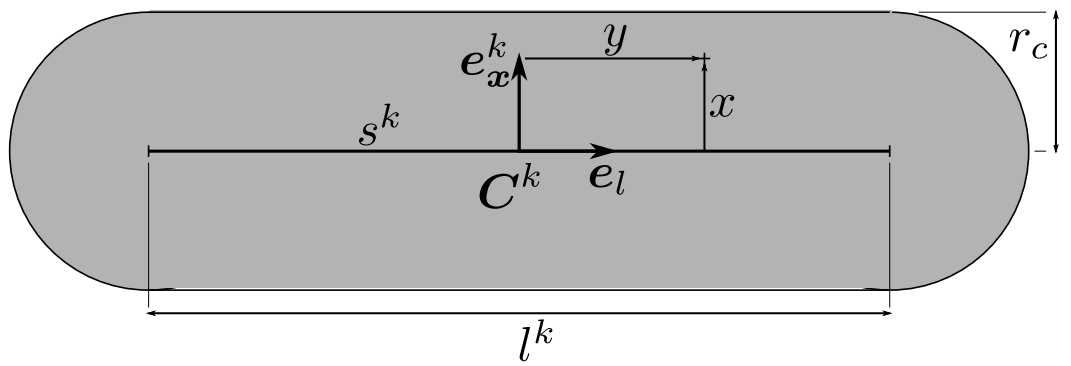

Figure 4: Local frame for the computation of $\mathscr{J}^{k}$ at point $\boldsymbol{x}$. The grey shaded area refers to the support of the function $\mathscr{J}^{k}$ in this local frame: if the point $\boldsymbol{x}$ is located outside this area, no eigenstrain will be attributed to it.

Thus $\boldsymbol{\sigma}^{F E}(\boldsymbol{x})$ at any point $\boldsymbol{x}$ is given by

$$
\boldsymbol{\sigma}^{F E}(\boldsymbol{x})=\int_{\mathbb{R}^{3}} \bar{w}\left(\left\|\boldsymbol{x}_{0}-\boldsymbol{x}\right\|\right) \tilde{\boldsymbol{\sigma}}^{F E}\left(\boldsymbol{x}_{0}\right) \mathrm{d} x_{0}^{3},
$$

where the notation $\mathrm{d} x_{0}^{3}$ denotes a volume integral. This integral is approximated numerically using the IP, as following:

$$
\boldsymbol{\sigma}^{F E}(\boldsymbol{x}) \simeq \frac{\sum_{j \backslash\left\|\boldsymbol{p}_{j}-\boldsymbol{x}\right\|<r_{c}} \bar{w}\left(\left\|\boldsymbol{p}_{j}-\boldsymbol{x}\right\|\right) \tilde{\boldsymbol{\sigma}}^{F E}\left(\boldsymbol{p}_{j}\right) V_{j}}{\sum_{j \backslash\left\|\boldsymbol{p}_{j}-\boldsymbol{x}\right\|<r_{c}} \bar{w}\left(\left\|\boldsymbol{p}_{j}-\boldsymbol{x}\right\|\right) V_{j}},
$$

where $\boldsymbol{p}_{j}$ is the position of IP $j$ and $V_{j}$ its associated volume.

\subsection{Interface handling}

In the DCM, the simulated volume $\Omega$ is delimited by periodic interfaces or by material interfaces. The treatments of those two kinds of interface differ and are described in this section.

\subsubsection{Periodic interfaces}

The dislocation dynamics and therefore the swept areas calculated with the DD code are by definition always located inside $\Omega$. When periodic boundary con- 
ditions are used and when a dislocation segment crosses a periodic interface, it reappears inside $\Omega$ modulo the linear dimensions of the simulated periodic volume. In the DD code the presence of a periodic medium is taken into account in a conventional way for the calculation of the local correction stress $\boldsymbol{\sigma}^{L C}$ and does need any special procedure for the DCM. Hence at each segment centre $\boldsymbol{\sigma}^{L C}$ is calculated considering the closest dislocation segments surrounding this point in the simulated periodic dislocation microstructure.

In the FE code a specific treatment is needed to compute the eigenstrain distribution near the boundaries of the FE mesh. Periodic replicas of those swept areas lying totally or partially at a distance smaller than the cut-off distance $r_{c}$ of the periodic FE mesh limits are created, and the regularisation procedure then takes these replicas into account in the same manner as the regular swept areas. Hence, a periodic dislocation microstrucure can exactly be taken into account. This additional procedure is illustrated in Fig. 5 in two dimensions. The process is the same in three dimensions: if a swept area lies in the $r_{c}$-vicinity of one periodic interface, it generates one replica, if it lies in the $r_{c}$-vicinity of two periodic interfaces, it generates three replicas, and if it lies in the $r_{c}$-vicinity of three periodic interfaces, it generates seven replicas.

Remark 3: Here again, the truncation of the stress fields computed by the DD code (equation (13)) provides large gains in computational cost as compared to the superposition method or to classical DD. Indeed, successive layers of periodic replicas of the simulated volume do not need to be considered anymore for the stress calculation, assuming that the simulated volume is big enough to contain a sphere of radius $r_{l}$. 


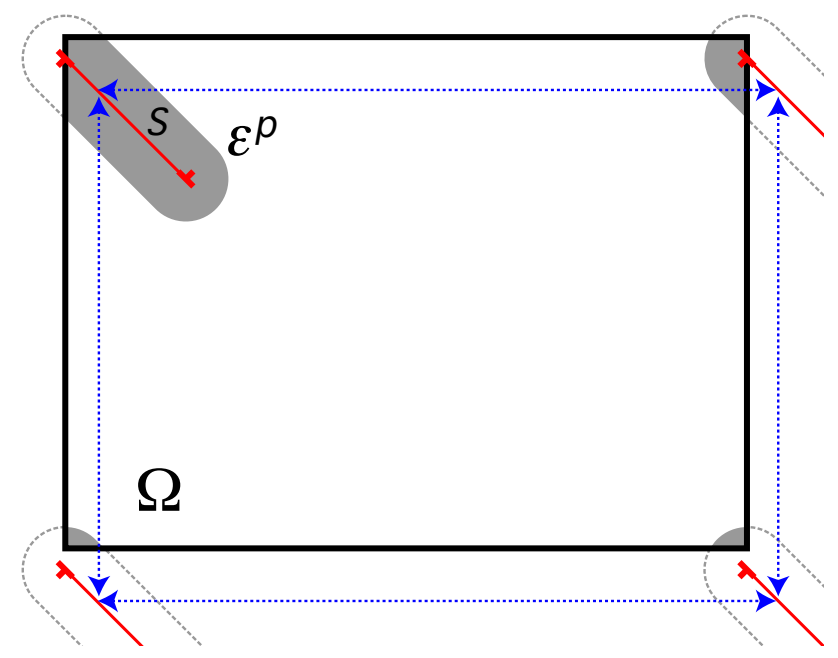

Figure 5: Two-dimensional example of the replicas of a glide area swept by a segment in the $r_{c}$-vicinity of the FE mesh boundaries (bold black lines). The blue arrows point to the periodic replicas that have to be generated to get a full periodic eigenstrain distribution $\boldsymbol{\varepsilon}^{p}$, which corresponds to the union of all grey areas.

\subsubsection{Material interfaces}

The problem of material interfaces in the DCM must be decomposed in two cases: those penetrable and those impenetrable by dislocations.

The problem of impenetrable interfaces is simple and no additional treatment is needed in the DCM procedures. More precisely, in the DD code the dislocation segments arriving at an impenetrable interface are simply blocked there. In the FE code, the slipped areas including those due to the dislocation segments within the vicinity of the interface are regularised only to those IP lying a the same side of the interface as the slipped areas but not beyond. In this case, the main numerical error apearing in DCM computations is limited to the close vicinity of interfaces and comes from the expressions for the local stress correction $\sigma^{L C}$ which do not account for the presence of two elastic media. The case of a free surface, denoted 
$\partial \Omega_{f} \subset \partial \Omega$, is less straightforward. Consider a dislocation loop whose dislocation line is denoted by $\ell$. Its movement generates a slipped area, denoted $A$, corresponding to the set of points in the slip plane delimited by $\ell$, i.e. $\ell$ is the boundary of $A$. The set of points $\mathscr{B}$ of the loop at the interface (forming a line segment or a poly-line segment) is then simply defined by $\mathscr{B}=A \cap \partial \Omega_{f}$.

When $\mathscr{B} \neq \emptyset$ different strategies can be considered. A first strategy depicted in Fig. 6-a would consist in arresting the dislocation line at the interface, as for the impenetrable case. This simple strategy has two main drawbacks. First, the DD code has to keep track of this part of the line which does not generate stress in the recomposed problem $\mathscr{P}$. Secondly, it introduces singular boundary terms $\left(\boldsymbol{u}^{S}-\boldsymbol{u}^{N S}\right.$ on $\partial \Omega_{u}, \boldsymbol{\sigma}^{S}-\boldsymbol{\sigma}^{N S}$ on $\left.\partial \Omega_{\sigma}\right)$ on a "large" surface of $\partial \Omega$ (see Fig. 7-e) which extends when more of $\ell$ is deposited on the interface.

The second strategy is depicted in 6-b. This time, when a section of $\ell$ reaches the interface, this line section is artificially moved outside the volume in its slip plane and in a direction normal to the line formed by the set of points $\mathscr{B}$ over a distance greater than $r_{c}$. The distance to which the dislocation should be moved outside is at least $r_{c}$, because any further away a dislocation is not seen any more by the IP of the FE mesh. In particular the swept area should be prolonged in the glide plane to reconstruct a continuous eigenstrain outside the simulated domain. For reasons of simplicity this procedure is currently only implemented for convex domains. This solution was found to remove the two drawbacks mentioned above: now the part of the extended dislocation line outside $\Omega$ has no mechanical influence anymore and so can be removed from the segment list in the DD code.

Then, as illustrated in Fig. 7-f, the only singular boundary terms introduced in the $\boldsymbol{\sigma}^{F E}$ problem solved with the FE solver are located at the points where 
the dislocation line crosses the interface. This is the strategy now adopted in the DCM. In addition, thanks to the localised requirement of the boundary terms $\left(\boldsymbol{u}^{S}-\boldsymbol{u}^{N S}\right.$ on $\partial \Omega_{u}, \boldsymbol{\sigma}^{S}-\boldsymbol{\sigma}^{N S}$ on $\left.\partial \Omega_{\sigma}\right)$ for the FE problem $\mathscr{P} F E$, the singular boundary terms can be neglected in many computations. Hence, the FE problem that is actually solved is

$$
\mathscr{P} F E=\begin{array}{ll}
\nabla \cdot \boldsymbol{\sigma}^{F E}+\boldsymbol{f}=\mathbf{0} & \text { in } \Omega \\
\boldsymbol{\sigma}^{F E}=\boldsymbol{E}:\left(\boldsymbol{\varepsilon}^{F E}-\boldsymbol{\varepsilon}^{p}\right) & \text { in } \Omega \\
\boldsymbol{u}^{F E}=\boldsymbol{u}_{0} & \text { at } \partial \Omega_{u} \\
\boldsymbol{\sigma}^{F E} \cdot \boldsymbol{n}=\boldsymbol{t} & \text { at } \partial \Omega_{\sigma} .
\end{array}
$$
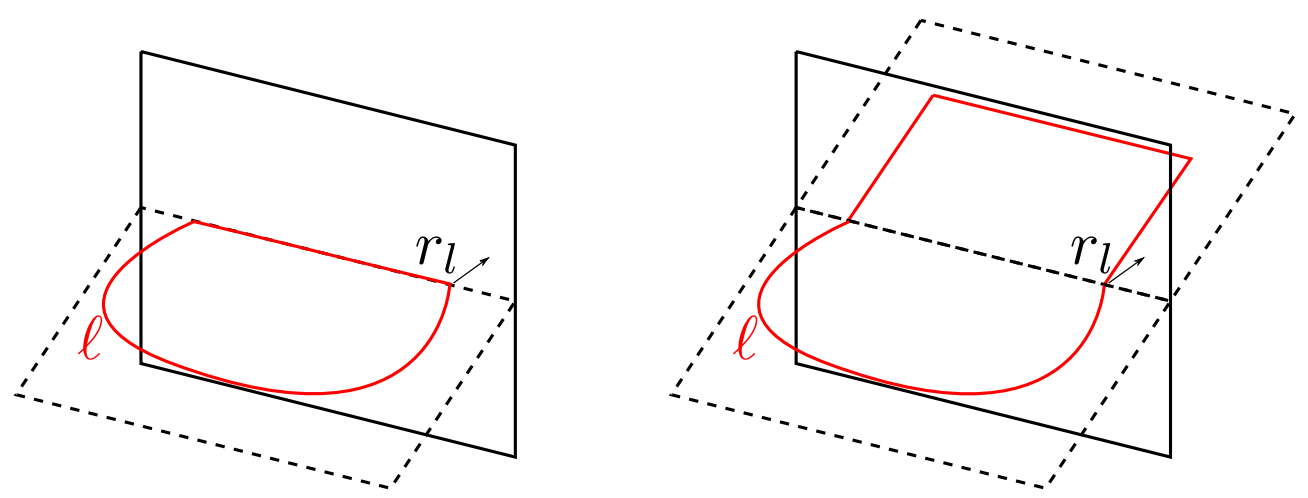

Figure 6: Illustration of the two possible strategies discussed in the text for handling the problem of a dislocation loop touching a penetrable interface. The red line is $\ell$. The blue shaded area is the part of $\partial \Omega$ on which boundary terms have to be introduced due to the part of $\ell$ that is at $\partial \Omega$ or outside $\Omega$.

\section{Improvement of numerical performance}

\subsection{Finite-element quadrature}

In the DCM the FE solver computes the stress $\boldsymbol{\sigma}^{F E}$, which is the FE approximation of the solution of the boundary value problem in $\Omega$ in the presence of the 
eigenstrain distribution, which is discretised to the IP by the regularisation procedure described previously. The choice of the element quadratures, roughly the number of IP per element, has a great influence on the computational costs and may affect the accuracy of the output $\boldsymbol{\sigma}^{F E}$ in quite a subtle way. Usually with FE computations, the richer the element quadrature, the better the FE solution approximates the continuum solution. This common prediction is not straightforward in the DCM as will be shown in this section.

When solving the boundary value problem with the FE method, plastic incompatibilities (the fact that the eigenstrain associated to dislocation slips cannot be written as the symmetric gradient of a displacement field) may lead to the generation of spurious stresses (called "eigenstress" in [24]) at two levels.

They can appear within an element, if there exists a sampling of the eigenstrain defined at the IP of the element which cannot be fitted by any function of the strain space of the element. This strain space refers to the vector space generated by the symmetrised gradient of the shape functions of the element. For example, consider a linear 4-node tetrahedron element with a 4-IP quadrature. In this element the strain is necessarily constant, whereas the sampling of an eigenstrain at its 4 IP is captured as non constant and then cannot be represented in the space of strain of the element. Elastic strain and its associated stress are then needed to accommodate the plastic strain. In the following we call this first kind of plastic incompatibility "intra-element" incompatibility.

Another type of plastic incompatibility may arise when two or more adjacent elements are distorted differently due to the plastic strain. Again elastic strain is needed to accommodate this incompatibility. In the following we call this second kind "inter-element incompatibility“. 
Through numerical investigation it appears that avoiding the intra-element incompatibility improves the quality of the FE solution. The removal of such intraelement plastic incompatibility may be performed using low-order quadrature, i.e. elements with a small number of IP. In the following, an element whose quadrature is such that any sampling of eigenstrain at its IP can be fitted by a function of its strain space is said to be self-compatible. The influence of the mapping from the reference element to the mesh element is here neglected: elements are considered to be homothetic to their reference element. For example, the quadratic 10-node tetrahedron with a 4-IP point quadrature, or the quadratic 20-nodes hexahedron with an 8-IP point quadrature (this element is under-integrated) are selfcompatible.

This assertion is now demonstrated with the simple test case of a straight infinite screw dislocation lying along the $z$-direction. The computational domain is a rectangular parallelepiped with periodic boundary conditions along the $z$ directions and free boundaries along the $x$ - and $y$-directions. The resulting stress field of this configuration can easily be calculated from existing analytical solutions [1]. The computational cell shown in Fig. 8 measures $L_{z}=100 \mathrm{~nm}$ in the $z$-direction and $L=800 \mathrm{~nm}$ in the other two directions. Isotropic elastic constant are used, with shear modulus $\mu=51 \mathrm{GPa}$ and Poisson ratio $v=0.37$ and the norm of the Burgers vector, oriented in the $z$-direction, is $b=0.25 \mathrm{~nm}$. The influence of the free boundaries is not commented here. Through the homogenisation procedure of $\S 2.2$, the movement of the dislocation from one edge of the computational cell along its slip plane generates a platelike inclusion shown in Fig. 8-a. The only non-zero component $\varepsilon_{y z}^{p}$ of the eigenstrain tensor is shown in Fig. 8-b. It can be observed that, except very close to the dislocation core, $\varepsilon_{y z}^{p}$ does not depend 
on $x$ inside the eigenstrain inclusion.

A regular structured FE mesh is used with $32 \times 32 \times 4$ quadratic hexahedra with 20 nodes and 27 IP. These IP are located on a regular $3 \times 3 \times 3$ grid inside each element. The mesh size is chosen such that the edge length of the elements equals the homogenisation length $h$. For this case, only one component of the resulting stress field is problematic: $\sigma_{y z}$. This component is shown (after convolution with $\bar{w}$ ) in Fig. 8-c. One can observe that instead of decreasing smoothly as $1 / r$ from the dislocation core as expected, $\sigma_{y z}$ shows artifacts along the $y$ axis that do not vanish away from the dislocation core. However, the solution of the continuous problem far from the dislocation core is $\left\{\boldsymbol{\sigma} \rightarrow \mathbf{0}, \boldsymbol{\varepsilon} \rightarrow \boldsymbol{\varepsilon}^{p}\right\}$, so the plastic strain should be compatible there.

These artefacts arising from the intra-element plastic incompatibilities can be understood as follows. Consider an element, far away from the dislocation core, with non-zero eigenstrain. Along the $y$-direction and passing through the IP, the eigenstrain $\varepsilon_{y z}^{p}(y)$ given by the regularisation procedure is a non-linear function (and far from the core, the problem is invariant in $x$ and $z$ ). The best-fit approximation given by the quadratic FE to this non-linear input $\varepsilon_{y z}^{p}(y)$ is linear, and this results in alternating positive and negative stresses $\sigma_{y z}^{F E}$ at the IP. Fortunately, if the intra-element incompatibilities are removed by using self-compatible quadratic hexahedra with 20 nodes and $8 \mathrm{IP}$, these oscillations of the stress $\sigma_{y z}^{F E}$ disappear, as shown in Fig. 8-d.

This first test is an idealised test case because all element edges are parallel and orthogonal to the slip plane of the dislocation. Then, far from the dislocation core, there are no inter-element plastic incompatibilities. A more general case is now considered where the element edges are not aligned with the slip plane of 
the dislocation by rotating the mesh with respect to the slip plane and the Burgers vector. Figures 9 give the results of this second case (again after convolution) for the 20-node hexahedron with 27 IP and the self-compatible 20-node hexahedron with 8 IP. Both solutions show spurious oscillations far from the the dislocation core. These oscillations result from both intra and inter-element incompatibilities for the non-self-compatible hexahedron, and only from inter-element incompatibilities for the self-compatible hexahedron. The solution for the 8-IP element is not only cheaper but appears to be slightly better. Naturally, the FE solution converges to the expected solution when refining the mesh for both the underintegrated element (allowing only inter-element plastic incompatibilities) and the fully-integrated element (allowing inter and intra-element plastic incompatibilities). Fig. 9-d shows the result for the self-compatible element for a finer mesh, with 4 elements across the homogenisation length. However for a given mesh, the FE solution is cheaper in terms of computational costs and at least as good with elements that do not allow intra-element plastic incompatibilities. The same conclusions can be given with 10-node 4-IP tetrahedra, as shown in the comparison of the $\boldsymbol{\sigma}_{x x}^{F E}$ component in Fig. 10.

As a conclusion of this section, it appears through numerical investigation that the choice of self-compatible elements, roughly with a small number of IP, offers a better compromise between accuracy and costs with respect to the choice of elements with high order quadratures.

\subsection{Time integration}

In this section the question of the numerical time integration scheme in the FE part is addressed, specifically with respect to the internal variable $\boldsymbol{\varepsilon}^{p}$.

In the DCM, the DD time step $\delta t$ is given by physical considerations and then 
is a fixed parameter of the algorithm for the integration of the evolution problem. As noted in $\S 2$, the total stress field $\boldsymbol{\sigma}=\boldsymbol{\sigma}^{F E}-\boldsymbol{\sigma}^{N S}+\boldsymbol{\sigma}^{S}$ can be decomposed into a remote stress field $\boldsymbol{\sigma}^{R}=\boldsymbol{\sigma}^{F E}-\boldsymbol{\sigma}^{N S}$ and a short-range stress field $\boldsymbol{\sigma}^{S}$. The remote stress field accounts for the interaction of the dislocations and boundaries lying at a distance $r>r_{l}$ to each point whereas the short-range stress field accounts for the dislocations lying at a distance $r<r_{l}$. Although the short-range part is very sensitive to small variations of segment positions due to its singularity, the remote part is much less sensitive to details of the spatial dislocation distribution in the microstructure. Therefore a larger time step $\Delta t$ can be used for updating the remote contribution of the stress field than for the short-range part. This is why the FE problem may be solved only every $\Delta t=m \delta t$, where $\delta t$ is the DD time step and $m \in \mathbb{N}$ is called the time step ratio.

Through the FE simulation, one has to integrate the evolution of the internal variable $\boldsymbol{\varepsilon}^{p}$ over each $\Delta t$ to get the plastic strain increment $\Delta \boldsymbol{\varepsilon}^{p}$. Historically an implicit integration scheme was used in the DCM [20, 22]:

$$
\boldsymbol{\varepsilon}^{p}(t+\Delta t)-\boldsymbol{\varepsilon}^{p}(t)=F\left(\Delta t, \boldsymbol{\varepsilon}^{p}(t+\Delta t)\right)
$$

where $F$ represents the DD simulation. This leads to a non-linear global FE system of equations which is solved using a quasi-Newton algorithm. The main drawback of this approach is that it is highly CPU consuming as at each iteration of the quasi-Newton scheme, the DD simulation of the whole $m$ DD time step matching the considered $\Delta t$ has to be carried out once again. Furthermore, at each iteration the stress needs to be interpolated to the dislocation segments.

Alternatively an explicit scheme is now proposed in the DCM:

$$
\boldsymbol{\varepsilon}^{p}(t+\Delta t)-\boldsymbol{\varepsilon}^{p}(t)=F\left(\Delta t, \boldsymbol{\varepsilon}^{p}(t)\right)
$$


where $\Delta \boldsymbol{\varepsilon}^{p}$ is computed explicitly and remains constant all along the FE time step. Therefore the global FE system becomes linear during each FE time step. Furthermore, the stiffness matrix is unchanged as long as the mesh and the topology of the Dirichlet boundary conditions are unchanged throughout the simulation. It can then be factorised once and for all. Practically, the explicit procedure to solve the DCM problem during $\Delta t=m \delta t$ is:

1. The stress field $\boldsymbol{\sigma}^{F E}$ is interpolated by means of a convolution (see $\S 2.1$ ) to the dislocation segments.

2. The DD code computes the non-singular part of the local correction $\boldsymbol{\sigma}^{N S}$ for each segment.

3. The DD code solves the dynamics of the dislocation segments for $m$ DD time steps $\delta t$, updating $\boldsymbol{\sigma}^{S}$ every $\delta t$ for each segment, but not $\boldsymbol{\sigma}^{R}$. When a segment is discretised in smaller segments or when segments are merged during the $m$ DD time steps, rules are defined in the DD code to interpolate $\boldsymbol{\sigma}^{R}$ for the new segments based on the nearest defined segment solution (usually the nearest connected segments along the dislocation line).

4. The eigenstrain increment $\Delta \boldsymbol{\varepsilon}^{p}$ is computed from the areas $\{\Delta A\}$ slipped during the $m$ DD time steps following the regularisation procedure presented in $\$ 2.2$.

5. The FE code solves its linear boundary value problem to get $\sigma^{F E}$ at $t+\Delta t$. The key steps of this procedure are represented schematically in Fig. 11. Generally, the drawback of using an explicit scheme is that it can become unstable and then requires very small time steps. However, in order to properly catch the contact interactions between the dislocation segments, the DD time step is constrained to values that are much smaller than the maximum time step ensuring FE 
stability. In other words, the time step is assumed to be precision-limited, and not stability-limited. In all the tests that were carried out, with DD time steps $\delta t$ based on physical considerations and time step ratio $m$ up to 100 , no significant instability has ever shown up. It can be noted that if problematic instabilities would show up in some simulations, using an implicit integrator for the short range interactions computed through the $m$ DD steps but keeping a simple forward Euler for the long range interactions computed by the FE code, as suggested in [35], would be possible.

\subsection{Data communication between FE and DD codes}

Data communication between the FE and DD codes has been optimized. Data exchange can be done either at the IP of the FE mesh, or at the segment integration points (SIP) for dislocation dynamics. In previous DCM versions all data exchange was done at the IPs and all homogenisation and localisation operations were done in the DD code. However for large FE meshes the number of IP is much larger than the number of SIP and so the data exchange is now done with respect to the SIP coordinates. On the one side, the DD code sends the locations of the SIP to the FE code, and the FE code interpolates its stress field there (through the convolution defined in §2.1) and sends it back to the DD code. On the other side, the DD code sends the areas swept by the moving dislocation segments to the FE code, from which the FE code computes the plastic strain at its IP following the homogenisation procedure explained in $\S 2.2$. In short, in Fig. 11 this means that the two central boxes Interpolation and Regularisation are now attached to the FE code instead of the DD code, as they were before. As a side effect this allowed clearing up many redundant data structures in our codes. 


\subsection{Proximity localisation}

Following the homogenisation procedure $(\$ 2.2)$, for each IP it has to be determined whether it lies at a distance $r>r_{c}$ from each segment, or not. If $r>r_{c}$ nothing has to be done. Otherwise, an increment of plastic strain has to be calculated for that IP. A brute-force approach would consist of computing the distance from each IP to each dislocation segment. This would require operations of the order of $O\left(n_{\text {seg }} \times n_{i p}\right)$, where $n_{\text {seg }}$ is the number of segments and $n_{i p}$ the number of IP, which may become prohibitive for large calculations. Fortunately, there exist more efficient solutions. This problem of efficiently determining which IP lie in the $r_{c}$-range of dislocation segments can be seen as $n_{s e g}$ classical range searching problems (see $[36,37]$ for a definition of the range searching problems and overviews of different solving techniques): for each moving segment $k$, one has to determine which IP lie in its range, defined as the portion of space $\Omega_{k}=\left\{\boldsymbol{x} \in \mathbb{R}^{3}\right.$ such that the distance from $\boldsymbol{x}$ to segment $k$ is smaller than $\left.r_{c}\right\}$. The techniques for solving this problem efficiently are based on a pre-processing of the cloud of points on which localisation information can be queried. For most of them, a tree data structure is constructed in which these points are spatially and hierarchically sorted.

In the DCM, each $n_{\text {seg }}$ range searching problem $\mathscr{P}_{k}$ is solved in two stages: first, a simpler problem $\mathscr{P}_{k}^{*}$ is solved by replacing $\Omega_{k}$ by its axis-aligned bounding box $\Omega_{k}^{*}\left(\mathscr{P}_{k}^{*}\right.$ falls in the category of orthogonal range searching problems). Then, the candidate IP inside $\Omega_{k}^{*}$ are checked one by one whether they actually belong to $\Omega_{k}$. The IP cloud is pre-processed at the beginning of the simulation into a "bounding volume hierarchy" whose bounding volumes are again axis-aligned bounding boxes. This pre-processing has to be carried out only once because 
remeshing is not yet done for DCM simulations, so it is unchanged throughout the simulation. Each problem $\mathscr{P}_{k}$ can then be solved with a complexity $O\left(\log \left(n_{i p}\right)\right)$. Thus the localisation of the IP with respect to the dislocation segments has a $O\left(n_{\text {seg }} \log \left(n_{i p}\right)\right)$ complexity, i.e. it is performed with high efficiency.

\section{Validation and performance tests}

In this section, several elementary validation tests are presented in order to demonstrate the results and the performance of the improved formulation of the DCM. Tests with more complex geometries and higher dislocation densities will be presented in a forthcoming paper.

\subsection{Validation tests}

This first elementary test aims at validating the modified local correction proposed in $\S 2$. The elastic fields of a infinite, straight single edge or screw dislocation lying along the $z$-direction were reconstructed using the DCM and compared to analytical solutions. The DCM stress field $\boldsymbol{\sigma}=\boldsymbol{\sigma}^{F E}+\boldsymbol{\sigma}^{N S}-\boldsymbol{\sigma}^{S}$ was expected to be as close as possible to the analytical singular stress field $\boldsymbol{\sigma}^{S}$. The geometry of the computational cell and the boundary conditions were the ones used in $\$ 3.1$. In this test the influence of the free boundaries was considered negligible because computed and analytic solutions for the dislocation stress field were compared sufficiently far away from the boundaries.

The computational cell measured $L_{z}=0.127 \mu \mathrm{m}$ in the $z$-direction and $L=$ $1.27 \mu \mathrm{m}$ in the other two directions. The crystal was assumed to be isotropic, with shear modulus $\mu=51 \mathrm{GPa}$ and Poisson ratio $v=0.37$. The norm of the Burgers vector was $b=0.25 \mathrm{~nm}$. The dislocation glided on a $y$-oriented plane from the $-x$-oriented face of the plate to the centre of the plate. For the FE problem, a 
structured mesh consisting of $80 \times 80 \times 8$ quadratic 20-node, 8 -IP hexahedra was used. For the regularisation, the homogenisation length was $h=125 b=31.9$ $\mathrm{nm} \approx L / 40$ and the cutting radius was $r_{c}=2 h=63.8 \mathrm{~nm} \approx L / 20$. The local correction radius was $r_{l}=2.5 h=79.8 \mathrm{~nm} \approx L / 16$.

2D color maps of the fields around the dislocation core are shown in Fig. 12. Only the non-zero components are represented. It can be observed that the absolute error $\boldsymbol{\sigma}-\boldsymbol{\sigma}^{S}$ is very small with respect to the amplitude of the stress fields. For the screw dislocation the maximum absolute error is located within the local correction area whereas for the edge dislocation, the maximum absolute error is located at $r=r_{l}$ where the DCM field shows some small discontinuities.

Figures 13 and 14 show graphs of the component $y z$ for the screw dislocation and $x y$ for the edge dislocation along the $x$ axis. The relative error with respect to the reference field $\boldsymbol{\sigma}^{S}$ is smaller than $5 \%$ for the screw dislocation and $8 \%$ for the edge dislocation. It is important to underline that the relative errors on the screw and on the edge component plotted in Fig. 12, Fig. 13 and 14 tend to zero as $r_{c}$ and $r_{l}$ increase. Here the values for $r_{c}$ and $r_{l}$ were chosen to find a good compromise between accuracy and performance of the DCM computations.

\subsection{Performance tests}

In order to test how the modifications presented in this paper really affect DCM performances, the duration of DCM time steps were compared with classical DD simulation time steps in a stress relaxation simulation.

A cubic simulation box with $L_{x}=L_{y}=L_{z}=5.0 \mu \mathrm{m}$ was chosen and periodic boundary conditions were applied. Calculations were run with three different initial dislocation densities: $0.9 \times 10^{12} \mathrm{~m}^{-2}$ (i.e. an average number of segments $n_{\text {seg }} \approx 2800$ during the calculation $), 1.0 \times 10^{13} \mathrm{~m}^{-2}\left(n_{\text {seg }} \approx 33000\right)$ and $5.2 \times$ 
$10^{13} \mathrm{~m}^{-2}\left(n_{\text {seg }} \approx 155000\right)$. No external loading was applied, so the dislocation dynamics was only driven by stress relaxation which includes many dislocationdislocation reactions.

In the DCM, for each dislocation density, three regular structured meshes were considered: $12 \times 12 \times 12$ elements with element edge $h_{e}=830 \mathrm{~nm}, 24 \times 24 \times 24$ with $h_{e}=415 \mathrm{~nm}$ and $48 \times 48 \times 48$ with $h_{e}=208 \mathrm{~nm}$. The time step ratio is set to 1 . In the classical DD simulations, for each dislocation density, the long range stress contribution was calculated with three different levels of approximation. Indeed, the exact solution of the periodic boundary conditions problem is formally difficult in 3D DD simulations and implies very intensive computations because an infinite number of periodic replicas would have to be taken into account. This problem does not exist in the DCM simulations because the periodic solution is easily obtained through the periodic displacement conditions applied in the elastic FE solver. In the DD simulations, the simulated volume is translated periodically by $n L_{x}, n L_{y}$ and $n L_{z}$ to give $n=0,1$ and 2 layers of replicas around the primary simulation box. Numerically, this translation is applied with the help of the FMM algorithm and therefore induces a only a moderate increase of the long-range stress field calculation in the classical DD simulations. More detail on the replica procedure and the multi-pole algorithm used in the DD simulation code microMegas can be found in [30]. The DD parts in both types of calculations were executed in parallel with MPI libraries using $12 \mathrm{CPU}$ cores for each simulation on a Intel Xeon X5670 machine, and the FE parts of the MDC calculations were executed on the same machine with 12 threads of the multithreaded FE solver.

The results of the performance tests are compared in Fig. 15. Several trends 
can be observed in this figure. First of all, as might be expected, among the classical DD simulations as the number of replicas and the number of segments increases, the time step increases. Of course, without replica layer the accuracy of the simulations should be called into question, but here the focus is only on the computation times. Then, for the DCM simulation an interesting trend can be observed. At the lowest number of segments, the calculation with the coarsest mesh goes faster, and the one with the finest mesh goes slower. However, going to higher number of segments, this trend is reversed: for the highest number of segments, the calculation with the finest mesh now is the fastest. This rather counter-intuitive result is explained as follows: for a low number of segments, most of the computational burden falls on the resolution of the linear system in the FE part of the DCM, whereas the time to calculate $\boldsymbol{\sigma}^{S}$ and $\boldsymbol{\sigma}^{N S}$ which is the most expensive part in the DD part, of order $O\left(n_{\text {seg }}^{2}\right)$, is needed for a relatively few interactions. Normally, at high number of dislocation segments, the computing time will be dominated by the latter part. However, for smaller mesh elements, the regions for which this analytical contribution has to be calculated can be made much smaller (because $r_{c}$ and $r_{l}$ are set to scale with $h$ and $h_{e}$ ). Even though the time needed for the resolution of the linear system in the FE part increases, this is more than offset by the decrease in the time to calculate $\boldsymbol{\sigma}^{S}$ and $\boldsymbol{\sigma}^{N S}$.

It is expected that this advantage will only hold up to a certain mesh refinement and that there is some optimum refinement. It is also expected that this optimum will be very sensitive to the computational performance and scalability of the FE solver. However these numerical problems are outside of the scope of the present article and have not been investigated yet.

Comparing the results of the DCM simulation to the classical DD simulation 
with the highest number of segments, it can be seen that the DCM calculation is almost as fast as the fastest classical DD simulation. Interestingly, even though the DCM was originally developed for finite domains, one must realize that in this case the DCM is more precise in periodic domains than the fastest classical DD simulation without replicas, and even faster already when one layer of replicas is taken into account in classical DD simulation. Finally, considering that the FE part essentially carries the long-range interactions (as well as the boundary conditions), it can be considered as an alternative for the multi-pole algorithm, where the cut-off distance between short-range and long-range interactions is now mathematically controlled by $r_{l}$.

\section{Conclusion}

A step forward in the simulation of the dynamical properties of large ensembles of dislocations is achieved. The Dicrete-Continuous Model (DCM), which couples a DD and a FE simulation code can now carry out realistic simulations over complex domains with large numbers of dislocations. More specifically, a consistent solution for the reconstruction of the short-range interactions has been developed. Furthermore, the DCM has been extended to unstructured meshes by adopting new efficient and accurate procedures for the regularisation of the slip by using a tree-based geometric algorithm for fast localisation. Also, a strategy for handling material interfaces or domain boundaries has been proposed. Some other aspects of the DCM, such as the time integration algorithm or the data communications between the two codes have been revised, and the choice of element type (interpolation order and number of integration points) has been justified. All these modifications lead to very significant gains in precision and speed. 
Ongoing calculations, to be published in a companion article, show that the progress made in the DCM method now allows for new types of simulations such as:

- Anisotropic elasticity simulation. In the particular case of structures for which a sufficiently refined mesh can be used (currently typically a few hundred nanometers) it is now possible to run DCM simulations without using the short-range stress field correction in the DD simulation code.

- Simulation of small micro-samples and micro-structured materials. The flexibility of the new DCM method allows modelling plastic size effects in materials with very complex boundaries and loading conditions. In short, it is now possible to track any problem that can be set up with a conventional small-strain FE method.

- Simulation of poly-crystalline or multi-domain microstructures. Multi-method simulations can be run with the DCM where the plastic deformation is calculated in a particular region with dislocation dynamics and in other regions with continuum constitutive laws.

\section{Acknowledgments}

The support of the research project Mera-FASS is acknowledged.

\section{References}

[1] J. P. Hirth, J. Lothe, Theory of Dislocations, MacGraw-Hill, New York, 1982. 
[2] B. Devincre, Three dimensional stress field expressions for straight dislocation segments, Solid State Communications 93 (1995) 875.

[3] W. Cai, A. Arsenlis, C. Weinberger, V. Bulatov, A non-singular continuum theory of dislocations, Journal of the Mechanics and Physics of Solids 54 (2006) 561-587.

[4] B. Devincre, A. Roos, S. Groh, Boundary problems in DD simulations, in: A. Finel, D. Mazière, M. Véron (Eds.), Thermodynamics, Microstructures and Plasticity, NATO Science Series, Kluwer Academic Publishers, Dordrecht, 2003, pp. 275-284.

[5] E. Van der Giessen, A. Needleman, Discrete dislocation plasticity: a simple planar model, Modelling and Simulation in Materials Science and Engineering 3 (1995) 689.

[6] M. Fivel, G. R. Canova, Developing rigourous boundary conditions to simulations of discrete dislocation dynamics, Modelling Simul. Mater. Sci. Eng. 7 (1999) 753-768.

[7] H. M. J.Zbib, T. Diaz de la Rubia, A multiscale model of plasticity, International Journal of Platicity 18 (2002) 1133-1163.

[8] D. Weygand, L. H. Friedman, E. van der Giessen, Aspect of boundary-value problem solutions with 3D dislocation dynamics, Modelling Simul. Mater. Sci. Eng. 10 (2002) 437-468.

[9] M. Tang, W. Cai, G. Xu, V. V. Bulatov, A hybrid method for computing forces on curved dislocations intersecting free surfaces in three-dimensional 
dislocation dynamics, Modelling and Simulation in Materials Science and Engineering 14 (2006) 1139-1151.

[10] K. W. Leiter, J. C. Crone, J. Knap, An algorithm for massively parallel dislocation dynamics simulations of small scale plasticity, Journal of Computational Science 4 (2013) 401-411.

[11] C. R. Weinberger, W. Cai, Computing image stress in an elastic cylinder, Journal of the Mechanics and Physics of Solids 55 (2007) 2027-2054.

[12] J. A. El-Awady, S. Bulent Biner, N. M. Ghoniem, A self-consistent boundary element, parametric dislocation dynamics formulation of plastic flow in finite volumes, Journal of the Mechanics and Physics of Solids 56 (2008) 2019-2035.

[13] A. Takahashi, N. M. Ghoniem, A computational method for dislocationprecipitate interaction, Journal of the Mechanics and Physics of Solids 56 (2008) 1534-1553.

[14] C. R. Weinberger, S. Aubry, S.-W. Lee, W. D. Nix, W. Cai, Modelling dislocations in a free-standing thin film, Modelling Simul. Mater. Sci. Eng. 17 (2009) 075007.

[15] S. Akarapu, H. M. Zbib, D. F. Bahr, Analysis of heterogeneous deformation and dislocation dynamics in single crystal micropillars under compression, International Journal of Plasticity 26 (2010) 239-257.

[16] J. A. El-Awady, S. I. Rao, C. Woodward, D. M. Dimiduk, M. D. Uchic, Trapping and escape of dislocations in micro-crystals with external and internal barriers, International Journal of Plasticity 27 (2011) 372-387. 
[17] C. Zhou, R. LeSar, Dislocation dynamics simulations of plasticity in polycrystalline thin films, International Journal of Plasticity 30-31 (2012) 185201.

[18] J. C. Crone, P. W. Chung, K. W. Leiter, J. Knap, S. Aubry, G. Hommes, A. Arsenlis, A multiply parallel implementation of finite element-based discrete dislocation dynamics for arbitrary geometries, Modelling and Simulation in Materials Science and Engineering 22 (2014) 035014.

[19] C. Lemarchand, B. Devincre, L. P. Kubin, J. L. Chaboche, Coupled mesomacro simulations of plasticity: Validation tests, in: V. Bulatov, T. Diaz de la Rubia, T. Phillips, R. andKaxiras, N. Ghoniem (Eds.), Multiscale Modelling of Materials, volume 538, MRS, Warrendale, Pennsylvania, 1999, pp. 6368.

[20] C. Lemarchand, B. Devincre, L. Kubin, Homogenization method for a discrete-continuum simulation of dislocation dynamics, J. Mech. Phys. Solids 49 (2001) 1969.

[21] Z. L. Liu, X. M. Liu, Z. Zhuang, X. C. You, A multi-scale computational model of crystal plasticity at submicron-to-nanometer scales, International Journal of Plasticity 25 (2009) 1436-1455.

[22] A. Vattré, B. Devincre, F. Feyel, R. Gatti, S. Groh, O. Jamond, A. Roos, Modelling crystal plasticity by $3 \mathrm{~d}$ dislocation dynamics and the finite element method: The discrete-continuous model revisited, Journal of the Mechanics and Physics of Solids 63 (2014) 491-505. 
[23] Y. Cui, Z. Liu, Z. Zhuang, Quantitative investigations on dislocation based discrete-continuous model of crystal plasticity at submicron scale, International Journal of Plasticity 69 (2015) 54-72.

[24] T. Mura, Micromechanics of Defects in Solids, Kluwer Academic Publishers, Dordrecht (The Netherlands), 1991.

[25] S. Groh, B. Devincre, L. Kubin, A. Roos, F. Feyel, J.-L. Chaboche, Dislocations and elastic anisotropy in heteroepitaxial metallic thin films, Phil. Mag. Letters 83 (2003) 303-313.

[26] Z. L. Liu, Z. Zhuang, X. M. Liu, X. C. Zhao, Z. H. Zhang, A dislocation dynamics based higher-order crystal plasticity model and applications on confined thin-film plasticity, International Journal of Plasticity 27 (2011) 201-216.

[27] S. Groh, B. Devincre, L. Kubin, A. Roos, F. Feyel, J.-L. Chaboche, Size effects in metal matrix composites, Materials Science and Engineering A 400-401 (2005) 279-282.

[28] A. Vattré, B. Devincre, A. Roos, Orientation dependence of plastic deformation in nickel-based single crystal superalloys: Discrete-continuous model simulations, Acta Materialia 58 (2010) 1938-1951.

[29] S. Groh, B. Devincre, F. Feyel, L. Kubin, A. Roos, J.-L. Chaboche, Discretecontinuum modeling of metal matrix composites plasticity, in: H. Kitagawa, Y. Shibutani (Eds.), IUTAM Symposium on Mesoscopic Dynamics of Fracture Process and Materials Strength, volume 115 of Solid Mechanics and its Applications, Kluwer Academic Publishers, NL-Dordrecht, 2004. 
[30] B. Devincre, R. Madec, G. Monnet, S. Queyreau, R. Gatti, L. Kubin, Mechanics of Nano-objects, Presses de l'Ecole des Mines de Paris, 2011.

[31] Z-set - non-linear material and structure analysis suite, 2015. URL: http://www.zset-software.com.

[32] J. Carrier, L. Greengard, V. Rokhlin, A fast adaptive multipole algorithm for particle simulations, SIAM Journal on Scientific and Statistical Computing 9 (1988) 669-686.

[33] H. Wang, R. LeSar, O(n) algorithm for dislocation dynamics, Philosophical Magazine A 71 (1995) 149-164.

[34] J. Yin, D. M. Barnett, S. P. Fitzgerald, W. Cai, Computing dislocation stress fields in anisotropic elastic media using fast multipole expansions, Modelling and Simulation in Materials Science and Engineering 20 (2012).

[35] R. B. Sills, W. Cai, Efficient time integration in dislocation dynamics, Modelling and Simulation in Materials Science and Engineering 22 (2014) 025003 .

[36] J. Bentley, J. Friedman, Data structures for range searching, ACM Computing Surveys (CSUR) 11 (1979) 397-409.

[37] P. Agarwal, J. Erickson, Geometric range searching and its relatives, Contemporary Mathematics 223 (1999) 1-56. 

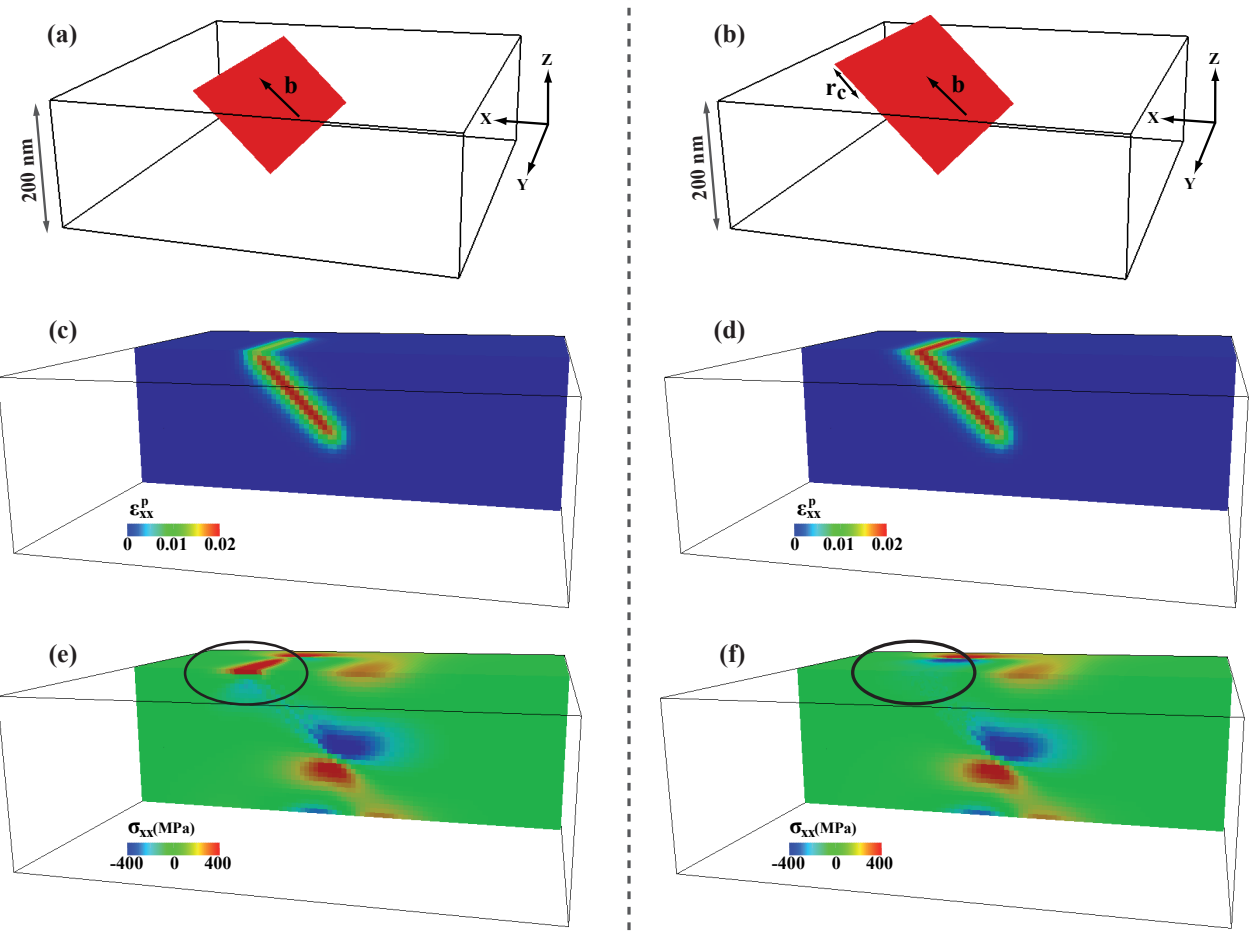

Figure 7: Application of the two strategies illustrated in Fig. 6 to the the problem of a dislocation loop emerging at a free surface. In this example $h=30 \mathrm{~nm}, r_{c}=1.75 h, b=0.25 \mathrm{~nm}$ and isotropic elastic constant are used, with shear modulus $\mu=51 \mathrm{GPa}$ and Poisson ratio $v=0.37$. (a) and (b) are the two swept areas corresponding to the strategy 1 or 2 . (c) and (d) are the corresponding eigenstrain field distributions. (e) and (f) are the $\boldsymbol{\sigma}_{x x}$ stress components calculated with the different strategies. Note the presence of artificial stresses at the surface of the solution (e). 

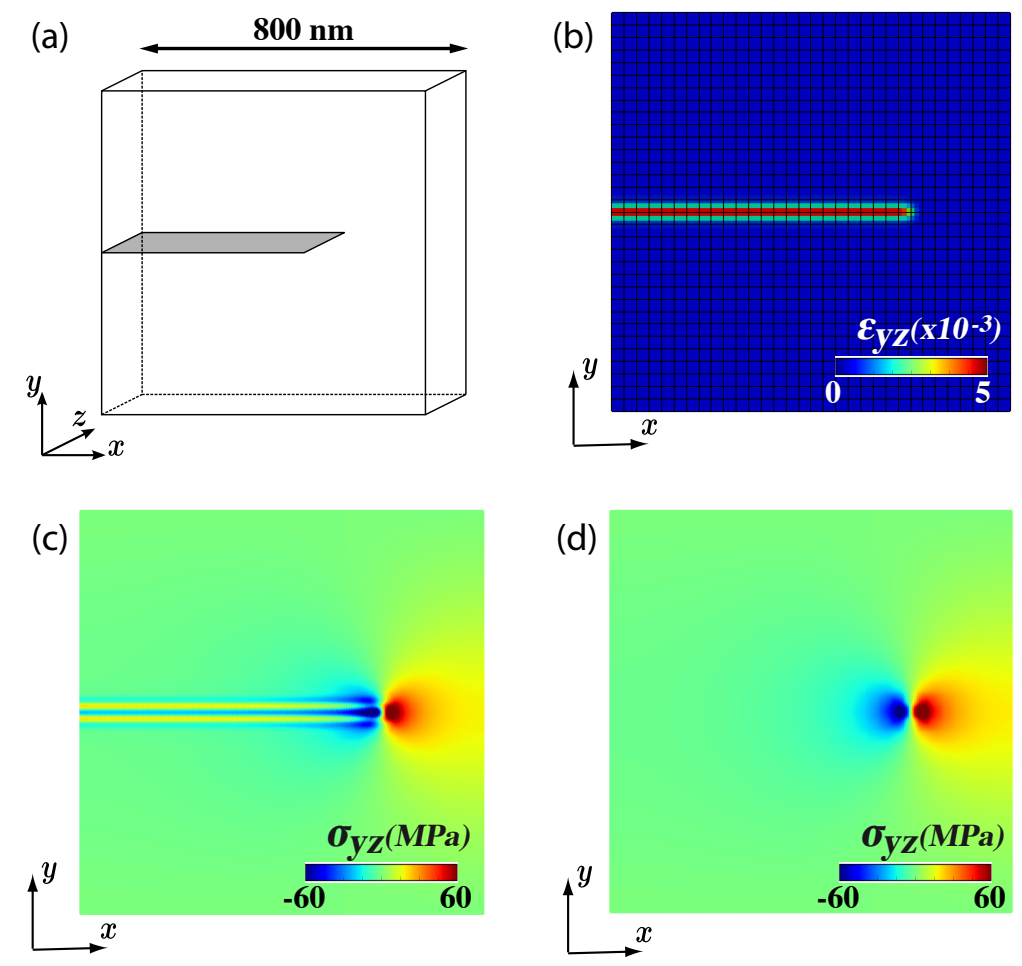

Figure 8: Finite element test aiming at reproducing the stress field of a single screw dislocation using the eigenstrain theory and the regularisation procedure of swept areas presented in paragraph 2.2. The swept area is shown in a), the resulting component of the eigenstrain $\varepsilon_{y z}^{p}$ and the $32 \times$ $32 \times 4$ mesh in b), $\sigma_{y z}^{F E}$ computed with 20-node and 27-IP elements in c) and $\sigma_{y z}^{F E}$ computed with 20-node and 8-IP elements in d). 
Figure 9: Same test as in Fig. 8 but with a mesh rotated with respect to the slipped area. $\varepsilon_{y z}^{p}$ and the mesh are given in a), $\sigma_{y z}^{F E}$ computed with 20-node, 27-IP elements in b), $\sigma_{y z}^{F E}$ computed with 20-node, 8-IP elements in c) and $\sigma_{y z}^{F E}$ computed with 20-node, 8-IP elements with a finer mesh (4 elements across $h$ ) in d). 

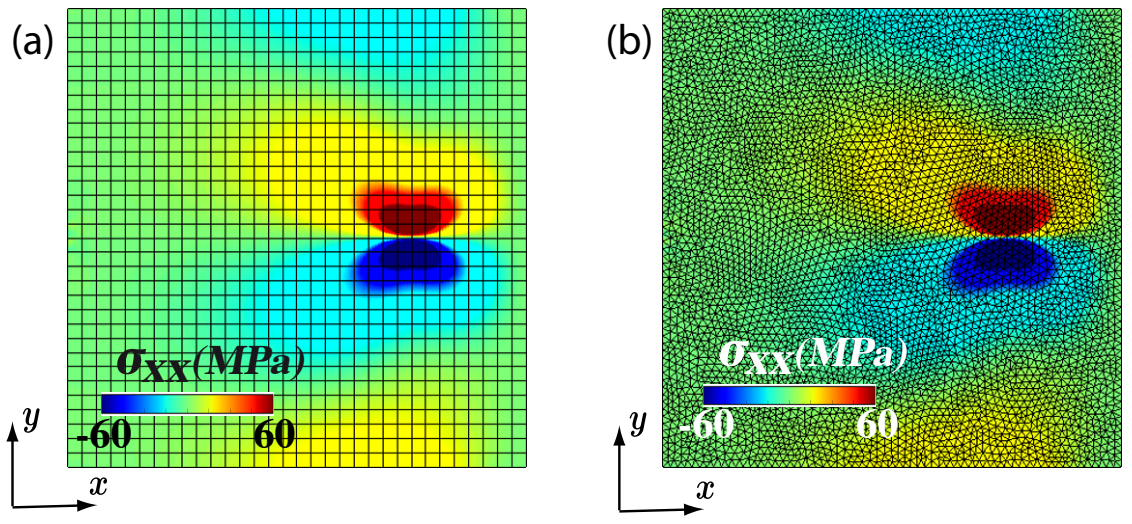

Figure 10: Comparison of $\sigma_{x x}^{F E}$ components computed with (a) a structured mesh made of 20node, 8-IP hexaedron elements and (b) an unstructured mesh made of 10-node, 4-IP tetrahedron elements. Both results correspond to the same configuration as shown in Fig. 8.

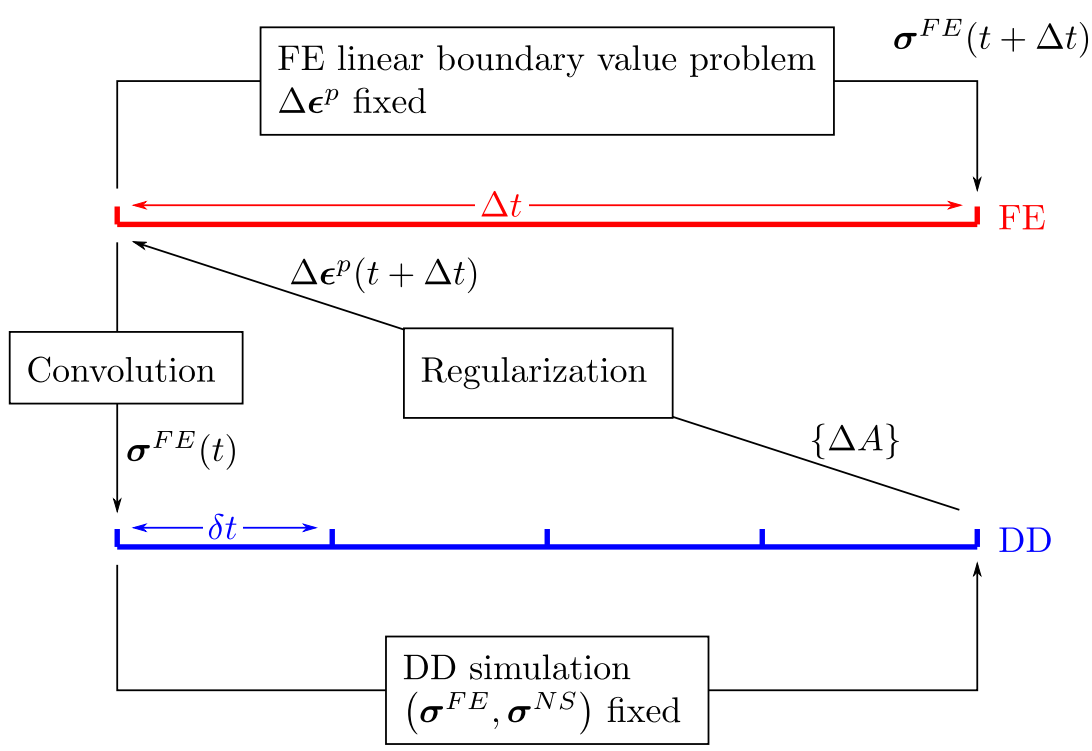

Figure 11: DCM: time integration algorithm. 

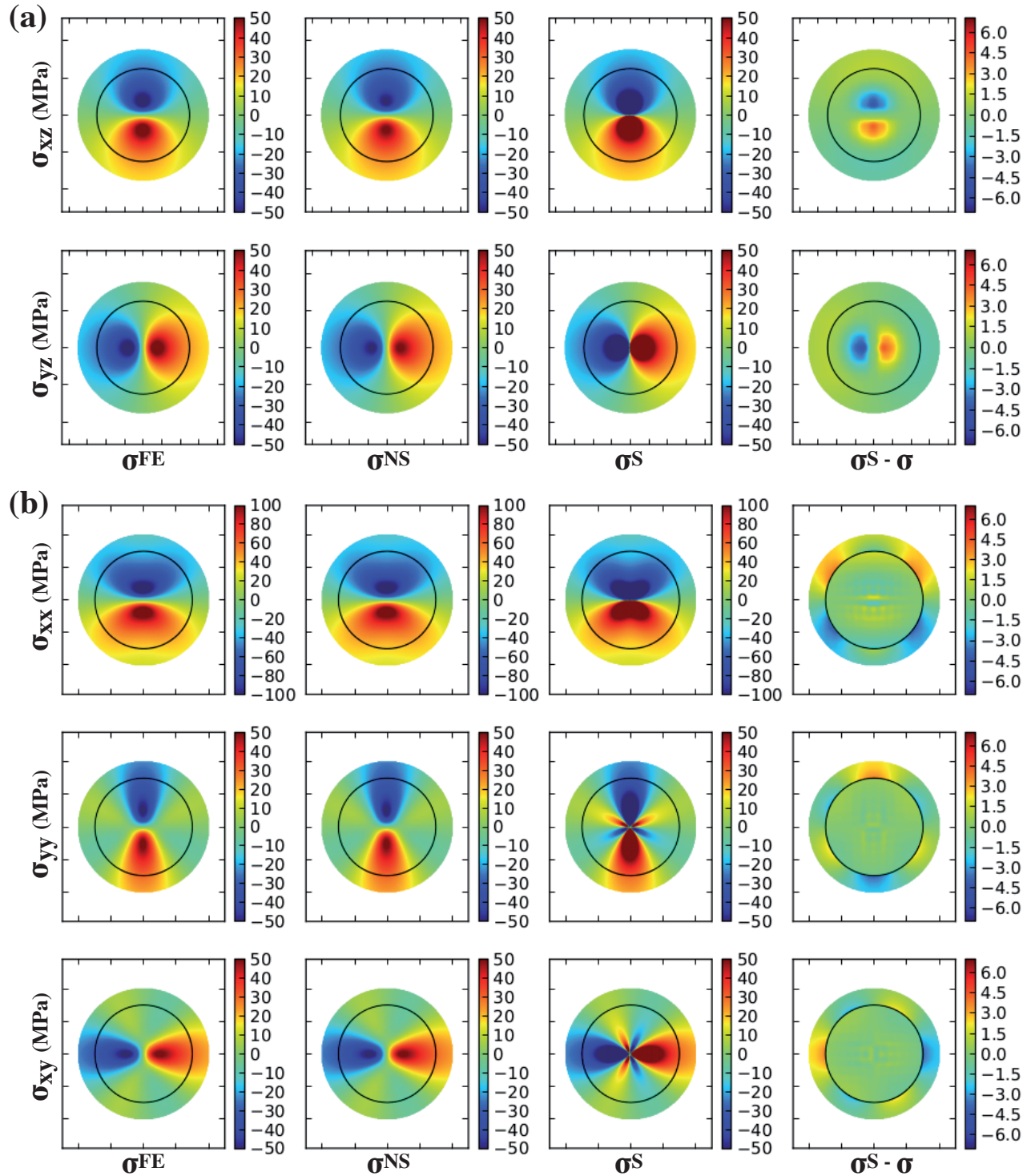

Figure 12: Non-zero components of the stress fields $\boldsymbol{\sigma}^{F E}, \boldsymbol{\sigma}^{N S}, \boldsymbol{\sigma}^{S}$ and of the absolute error $\boldsymbol{\sigma}-\boldsymbol{\sigma}^{S}$ around the core of a straight, infinitely long (a) screw, and (b) edge dislocation. The black circle delimits the local correction area at $r_{l} \approx L / 16$ around the dislocation core. 


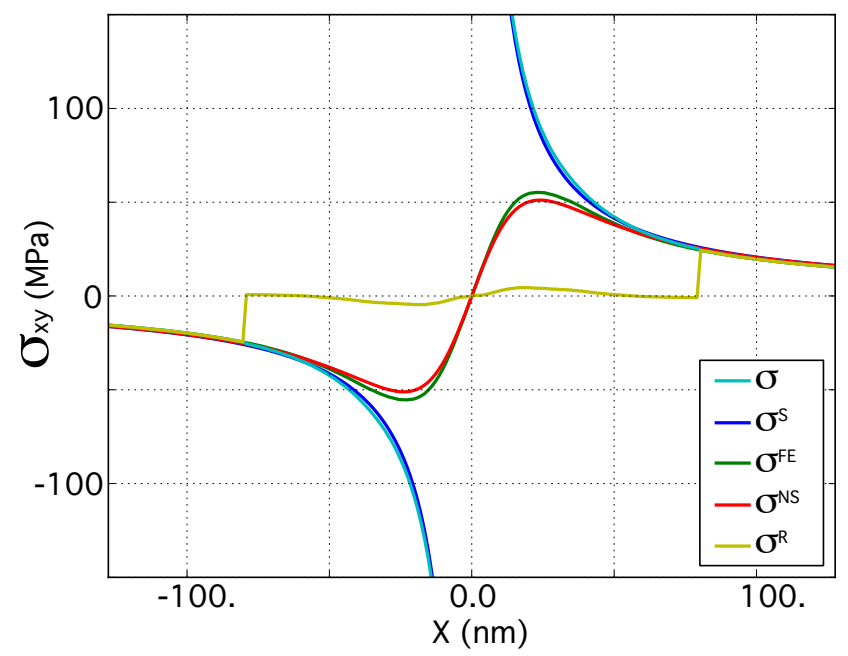

Figure 13: (a) $\sigma_{x y}$ components of $\boldsymbol{\sigma}, \boldsymbol{\sigma}^{S}, \boldsymbol{\sigma}^{F E}, \boldsymbol{\sigma}^{N S}$ and $\boldsymbol{\sigma}^{R}$ along the $x$-axis for the infinitely long straight screw dislocation.

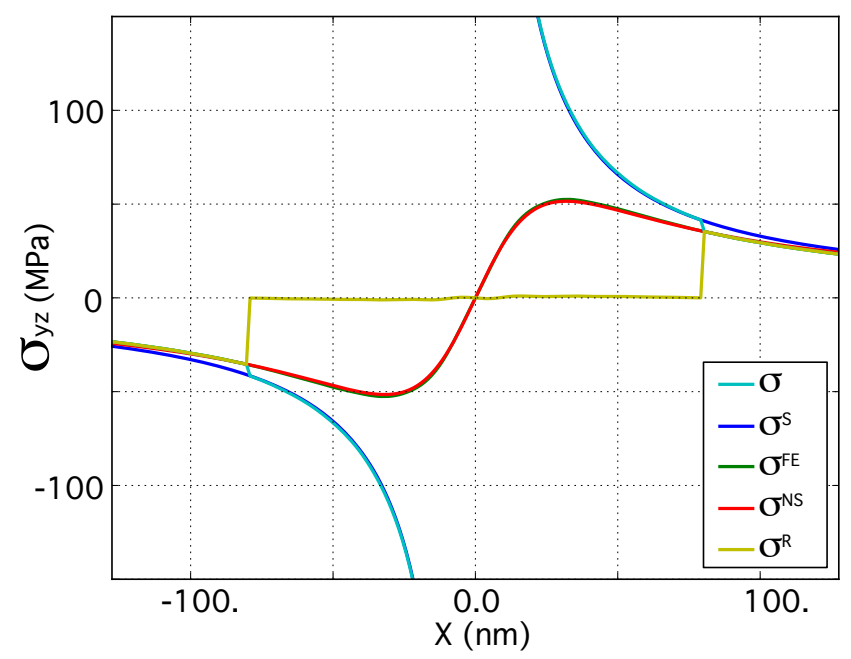

Figure 14: (a) $\sigma_{y z}$ components of $\boldsymbol{\sigma}, \boldsymbol{\sigma}^{S}, \boldsymbol{\sigma}^{F E}, \boldsymbol{\sigma}^{N S}$ and $\boldsymbol{\sigma}^{R}$ along the $x$-axis for the infinitely long straight edge dislocation. 


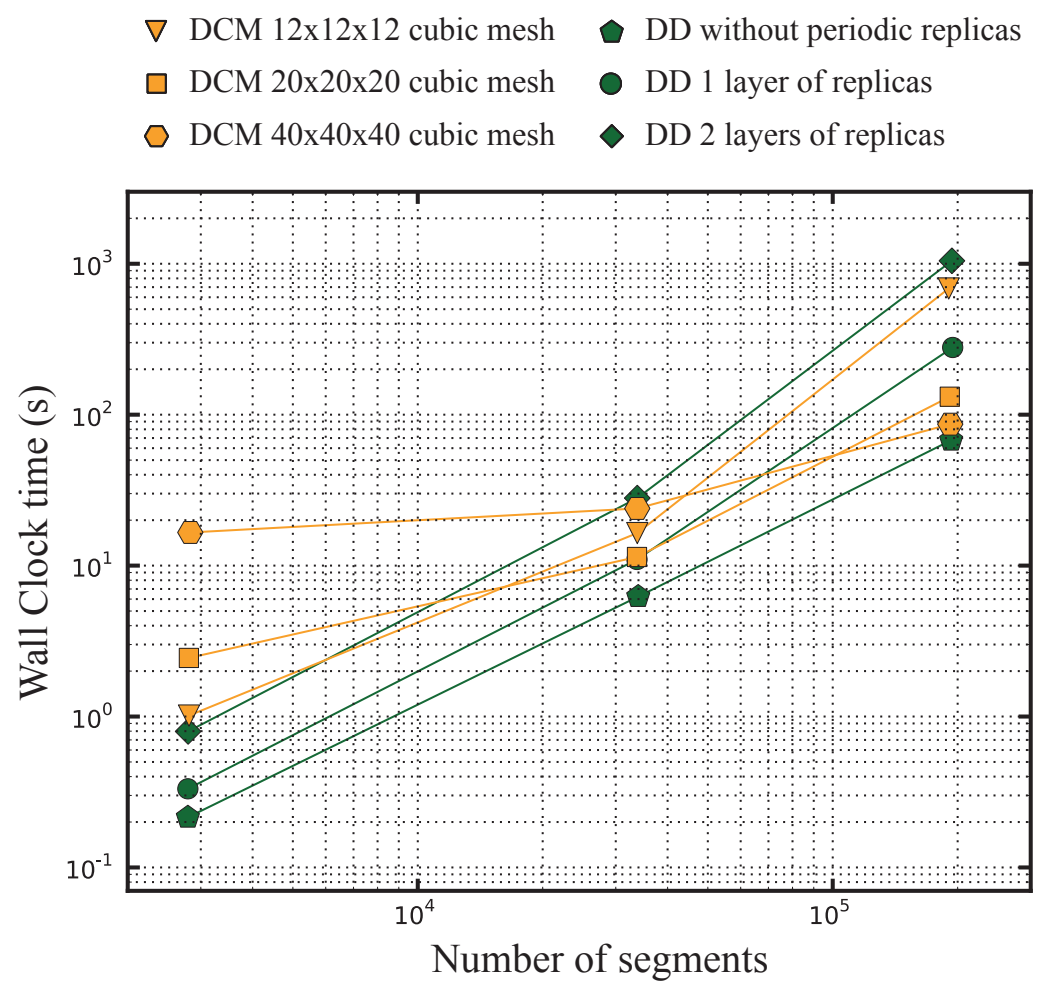

Figure 15: Performance tests (wall clock time per time step $\Delta t=\delta t$ as a function of the number of segments $n_{\text {seg }}$ in the simulation). In the DCM simulations (yellow symbols) three different mesh refinements were used and in the classical DD simulations (green symbols) the number of layers of replicas around the simulation cell increased from 0 to 2 . 


\section{Regularization}

\section{$\epsilon^{p}$}

FE boundary value problem solving

\section{Dislocation Dynamics}

$$
\{A\}
$$




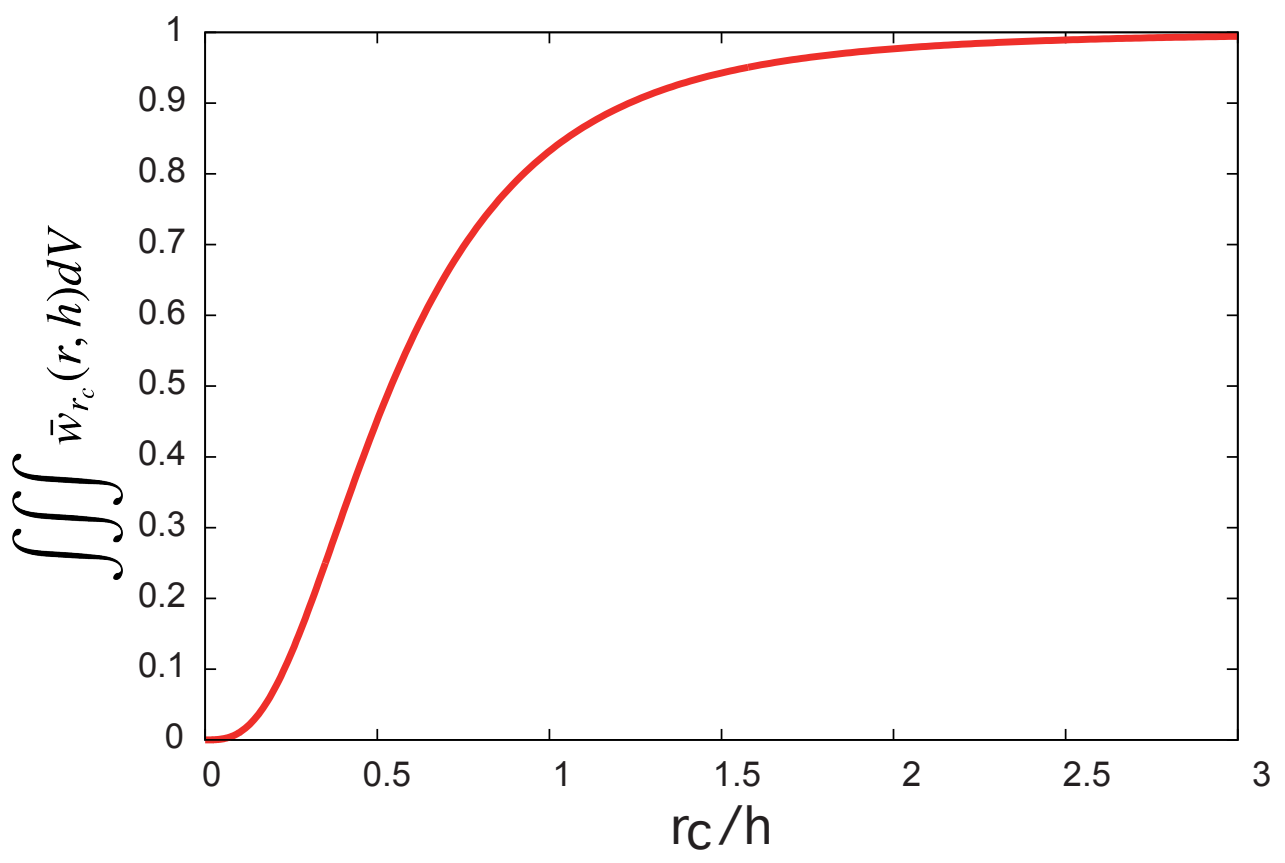




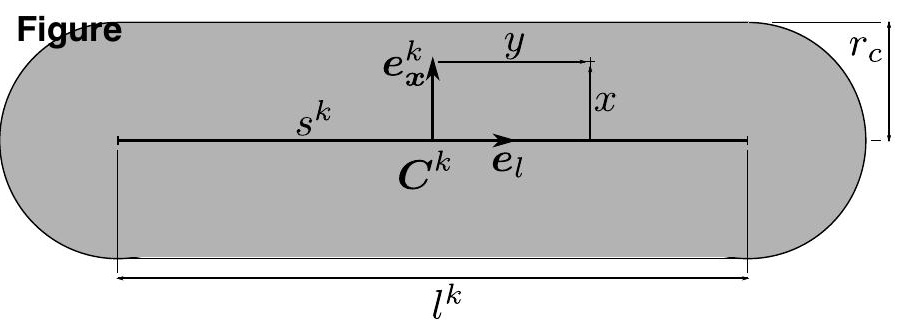


Figuiro

$\varepsilon^{p}$

$\Omega$

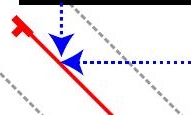




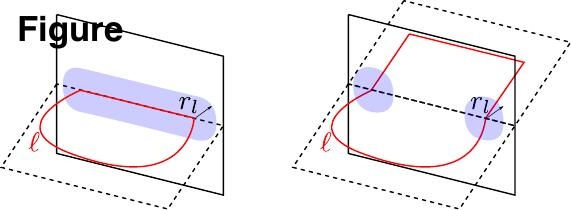




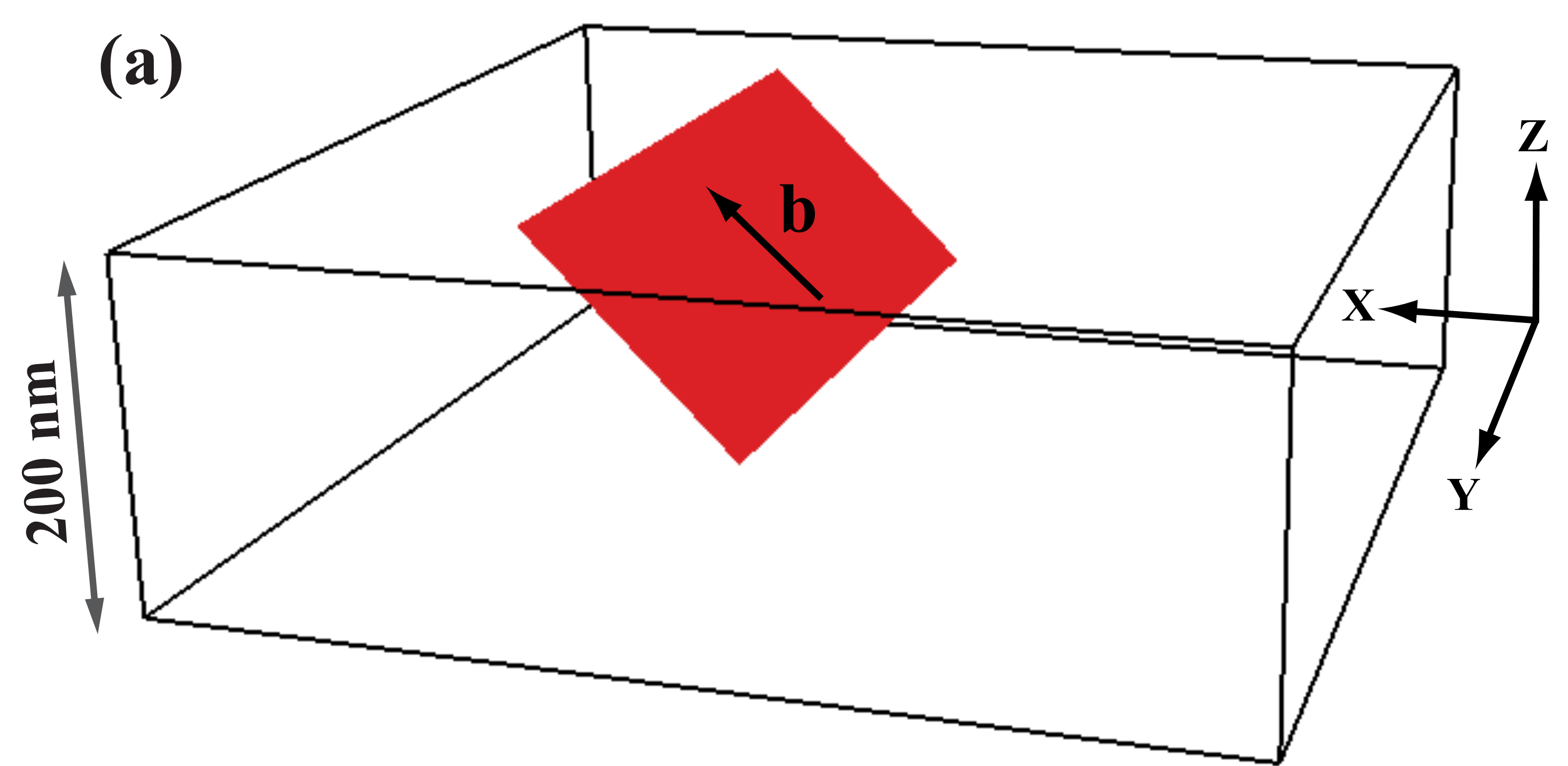

(c)

$$
\begin{array}{lll}
\multicolumn{2}{|c|}{\varepsilon_{\mathrm{xx}}^{\mathrm{p}}} \\
\mathbf{0} & 0.01 & 0.02 \\
\hline
\end{array}
$$

(e)

$$
\sigma_{\mathrm{xx}}(\mathrm{MPa})
$$$$
\begin{array}{lll}
-400 & 0 & 400
\end{array}
$$

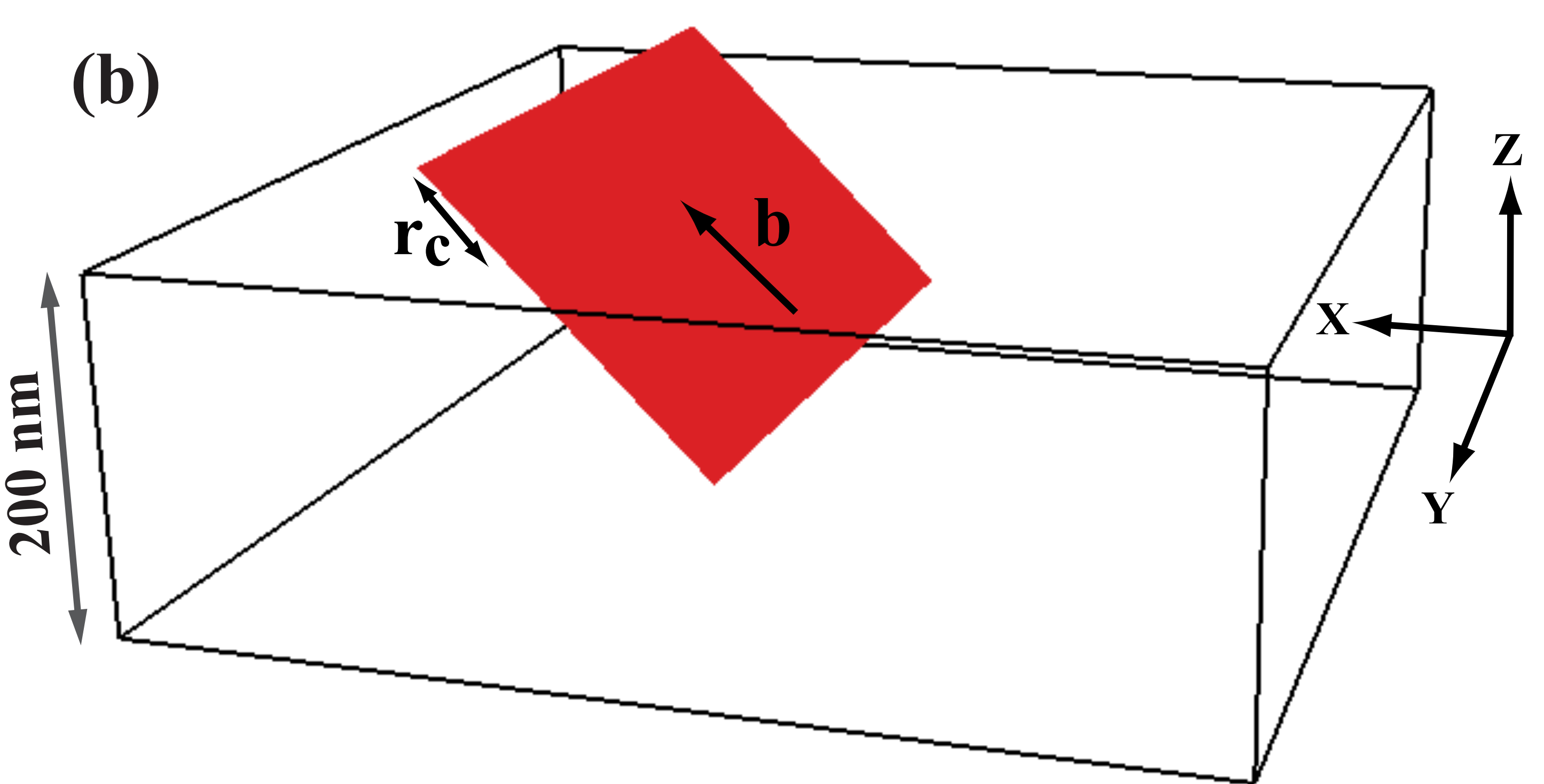

(d) \begin{tabular}{lll}
$\varepsilon_{\mathrm{xx}}^{\mathrm{p}}$ & \\
\hline $\mathbf{0}$ & 0.01 & 0.02
\end{tabular}

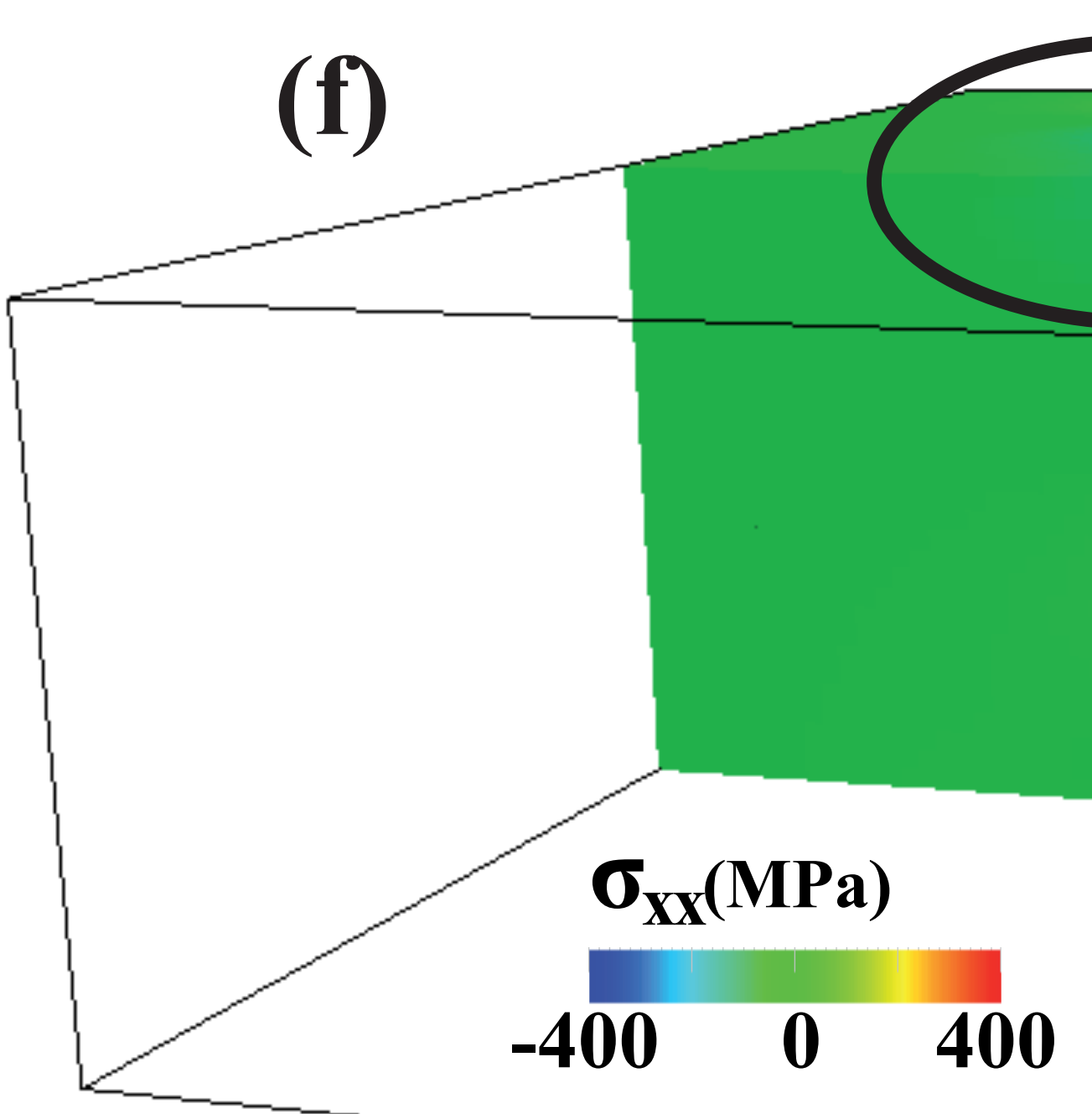


Figure

(a)

(b)

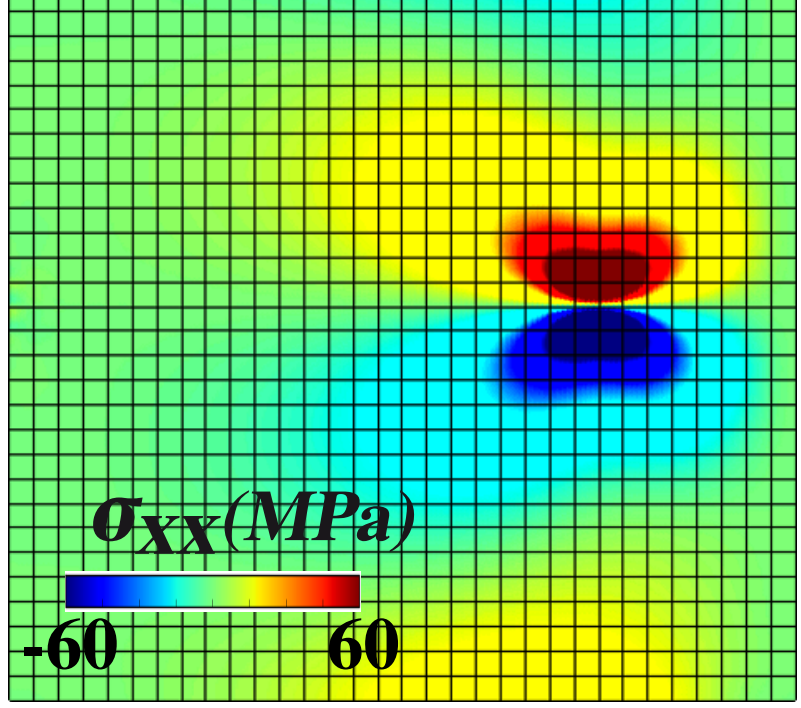

$x$

$4^{y}$

3.

71

W W $x$ 

$\Delta \boldsymbol{\epsilon}^{p}$ fixed

$\boldsymbol{\sigma}^{F E}(t+\Delta t)$

Convolution

$\Delta t$

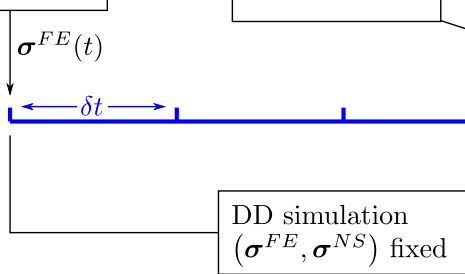


Figure
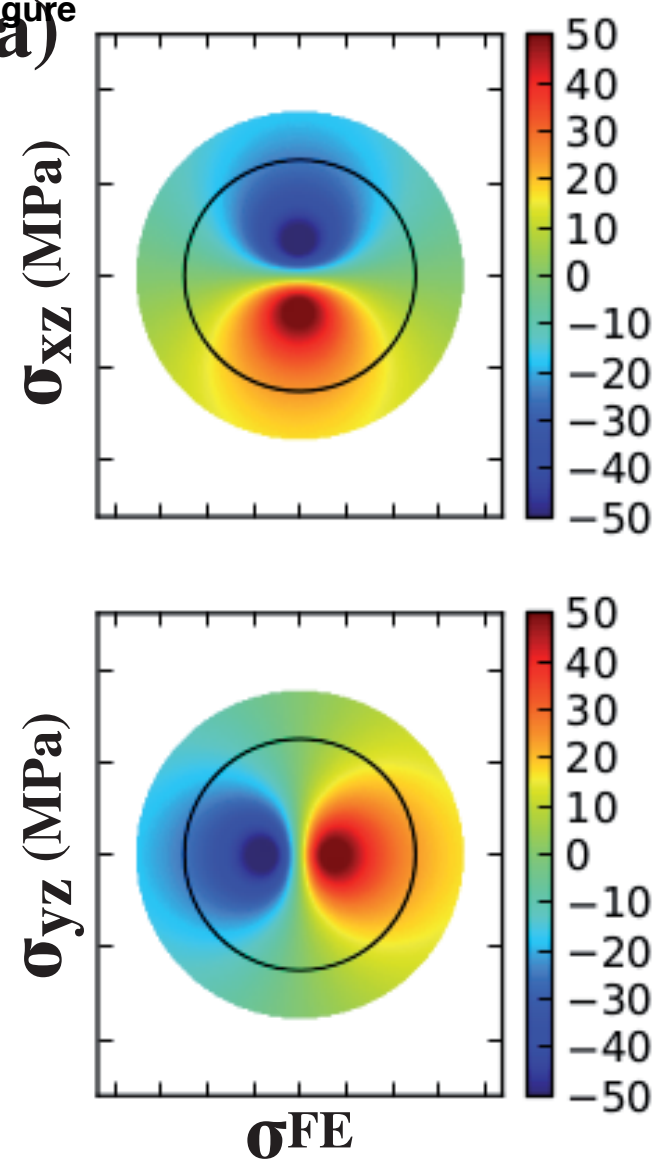

(b)
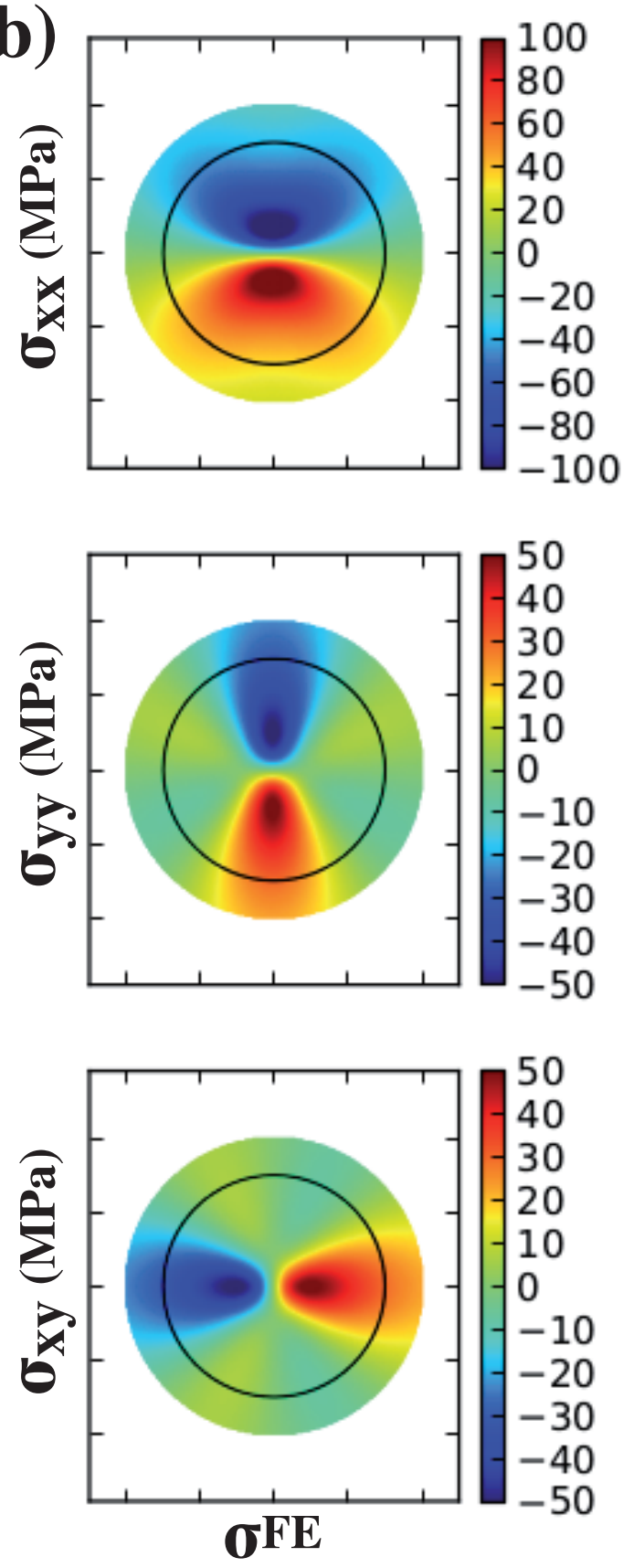
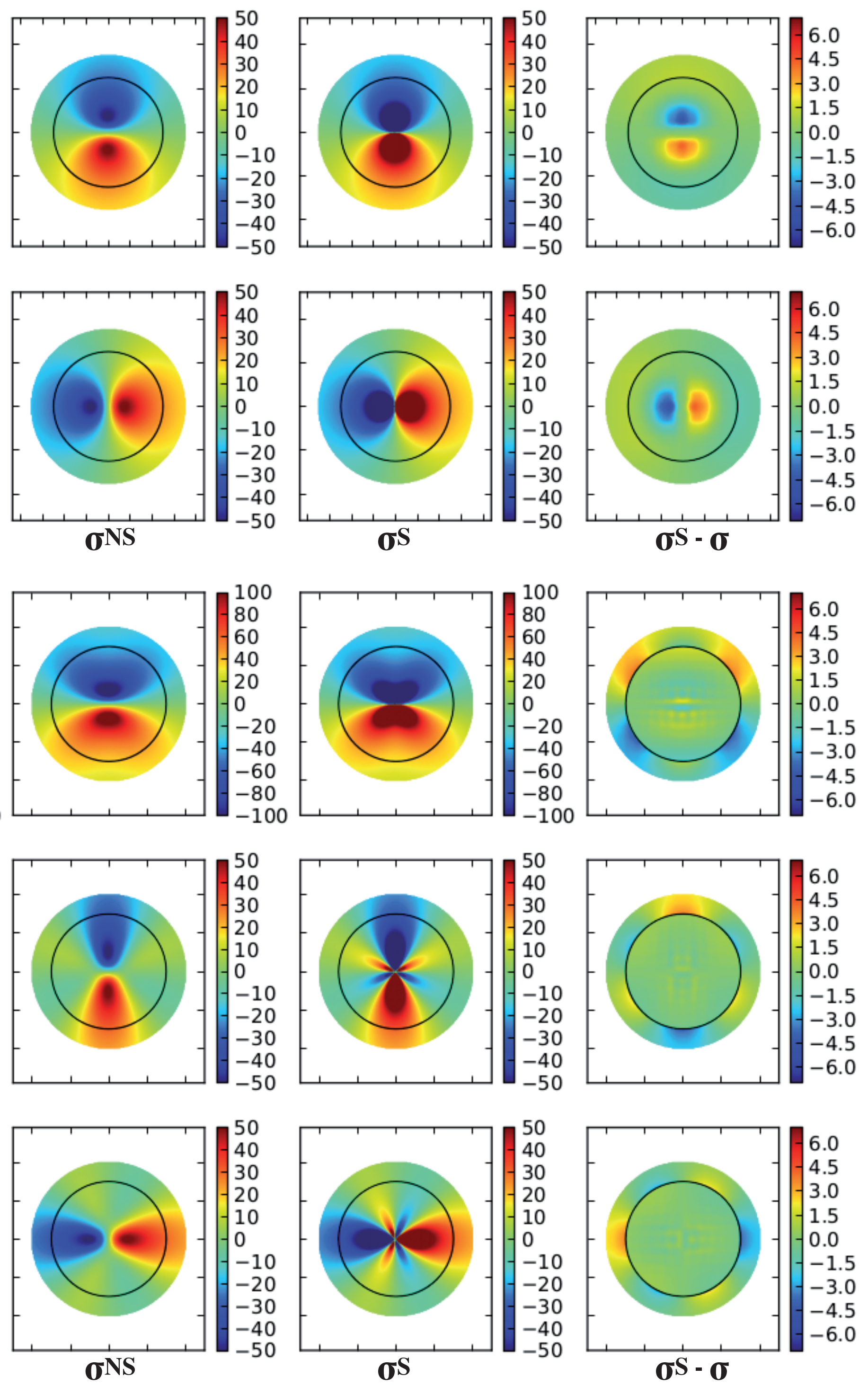
Figure

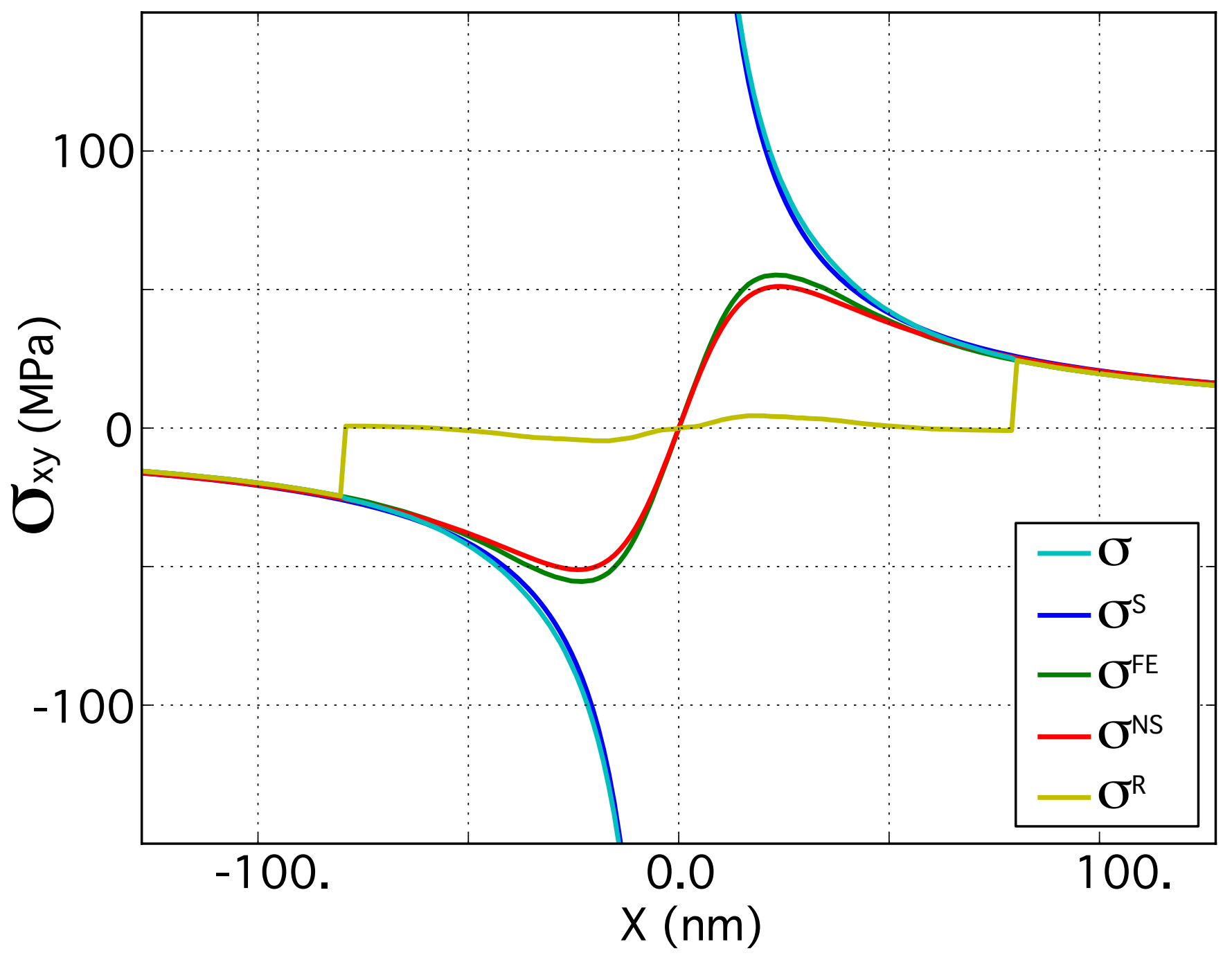


Figure

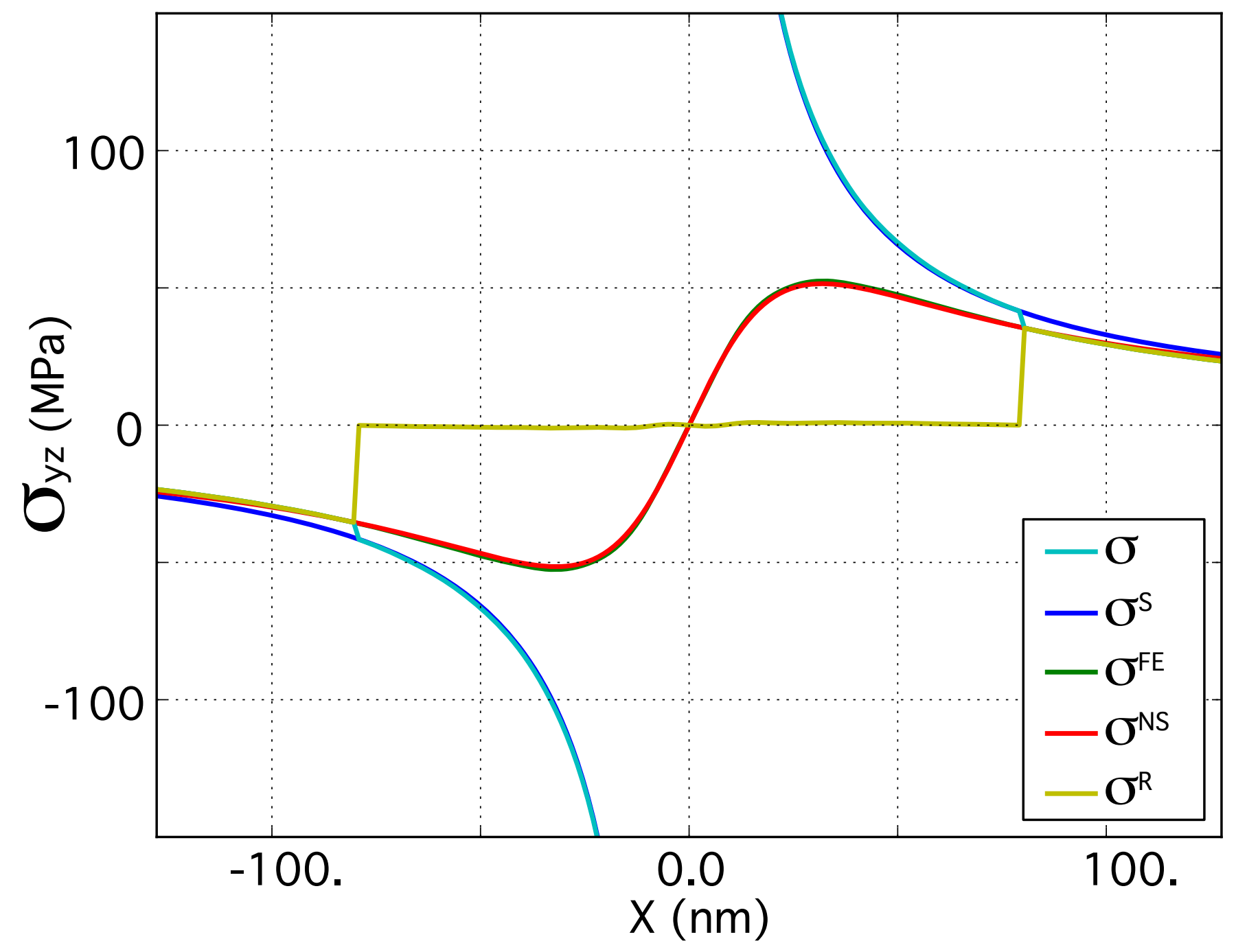


$\nabla$ DCM $12 \times 12 \times 12$ cubic mesh

DCM 20x20x20 cubic mesh

- DCM 40x40x40 cubic mesh
DD without periodic replicas

- DD 1 layer of replicas

$\diamond$ DD 2 layers of replicas

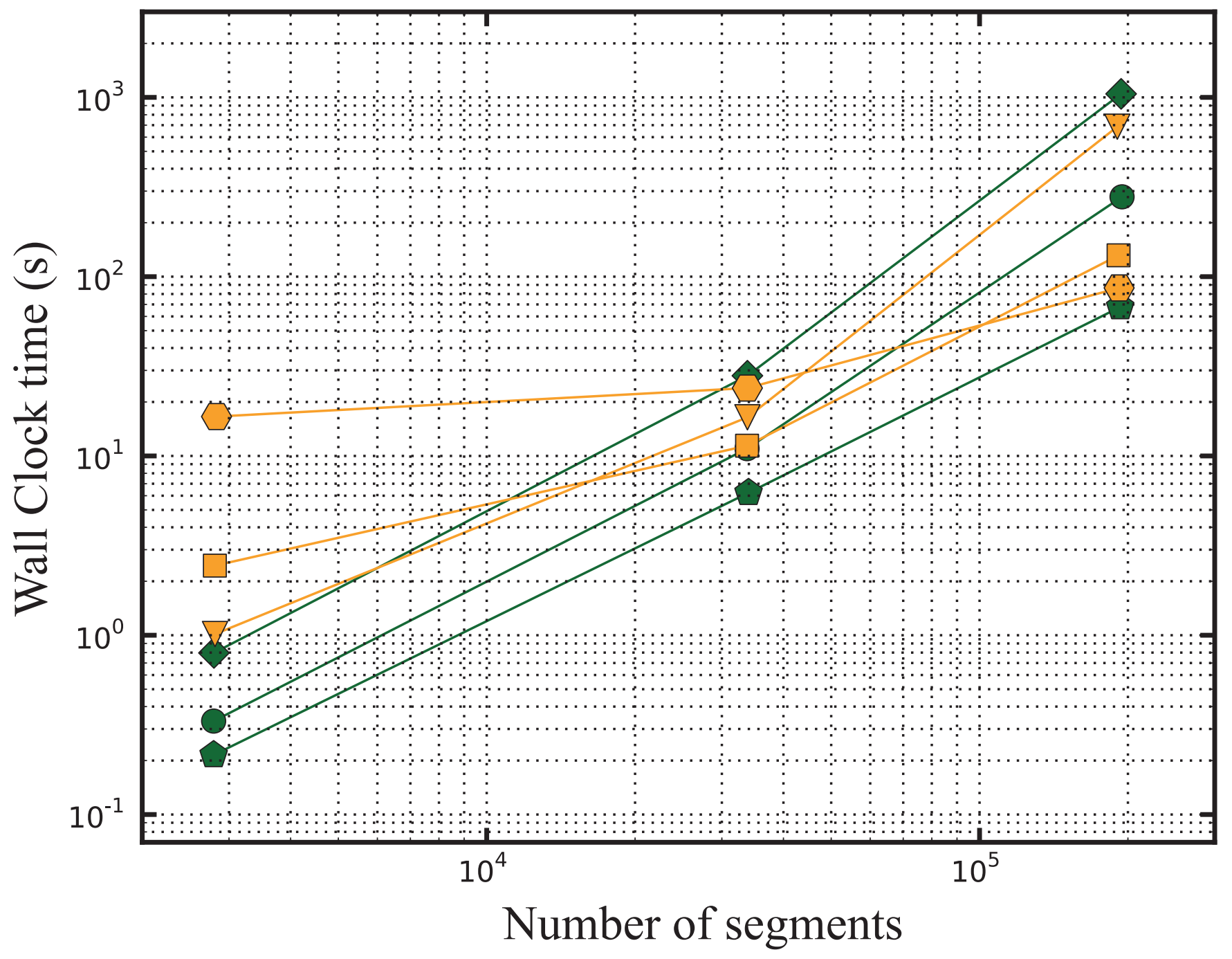

